ITP-SB-93-17

hep-th/9304150

\title{
Virasoro Characters from Bethe Equations for the Critical Ferromagnetic Three-State Potts Model
}

\author{
Srinandan Dasmahapatra, \\ High Energy Physics, ICTP, P.O. Box 586, I-34100, Trieste, Italy \\ Rinat Kedem, Barry M. McCoy, and Ezer Melzer \ \\ Institute for Theoretical Physics, SUNY, Stony Brook, NY 11794-3840, USA
}

\begin{abstract}
We obtain new fermionic sum representations for the Virasoro characters of the confromal field theory describing the ferromagnetic three-state Potts spin chain. These arise from the fermionic quasi-particle excitations derived from the Bethe equations for the eigenvalues of the hamiltonian. In the conformal scaling limit, the Bethe equations provide a description of the spectrum in terms of one genuine quasi-particle, and two "ghost" excitations with a limited microscopic momentum range. This description is reflected in the structure of the character formulas, and suggests a connection with the integrable perturbation of dimensions $(2 / 3,2 / 3)^{+}$ which breaks the $S_{3}$ symmetry of the conformal field theory down to $\mathbf{Z}_{2}$.
\end{abstract}

\footnotetext{
${ }^{1}$ rinat or mccoy or melzer @max.physics.sunysb.edu
} 


\section{Introduction}

The critical three-state Potts model was found to be integrable over 20 years ago [1, 2], and since these initial investigations it has been the subject of many studies [3]. Recently [4], it was shown that the order one excitations of the anti-ferromagnetic three-state Potts spin chain [5], computed from the formalism of functional and Bethe equations [6]-[12], can be used to construct expressions for the characters of the conformal field theory of $\mathbf{Z}_{4}$-parafermions. Since these equations yield excitations which obey a fermionic exclusion rule, we call these fermionic sum representations. These character formulas were previously obtained by Lepowsky and Prime [13] from considerations of the representation theory of the affine Lie algebra $A_{1}^{(1)}$. The characters, which in this case are branching functions of $\left(A_{1}^{(1)}\right)_{4} / U(1)$, are the building blocks of the modular invariant partition function of the conformal field theory.

Here we provide a parallel discussion for the ferromagnetic chain, leading to fermionic sum representations for the Virasoro characters [14] of the $\mathbf{Z}_{3}$-parafermionic conformal field theory which is associated with this model [15, 16, 17]. These representations, which we will now summarize, are quite different from the ones of [13].

The normalized Virasoro characters $\widehat{\chi}_{\Delta} \equiv q^{\frac{1}{30}-\Delta} \chi_{\Delta}$ of the $\mathbf{Z}_{3}$-parafermionic conformal field theory, with central charge $c=\frac{4}{5}$ and conformal dimensions $\Delta=\Delta_{r, s}=\frac{(6 r-5 s)^{2}-1}{120}$ $(r=1,2,3,4, s=1,3,5)$, are given by 14

$$
\widehat{\chi}_{\Delta_{r, s}}(q)=\widehat{\chi}_{\Delta_{5-r, 6-s}}(q)=\frac{1}{(q)_{\infty}} \sum_{k=-\infty}^{\infty}\left[q^{k(30 k+6 r-5 s)}-q^{(5 k+r)(6 k+s)}\right] .
$$

Our result here is that these characters can be written in the form

$$
\widehat{\chi}_{\Delta}(q)=\sum_{\substack{m_{1}, m_{2}, m_{3}=0 \\
\text { restrictions }}}^{\infty} q^{\frac{1}{4} \mathbf{m} C_{A_{3}} \mathbf{m}^{t}-\frac{1}{2} L(\mathbf{m})} \frac{1}{(q)_{m_{1}}}\left[\begin{array}{c}
\frac{1}{2}\left(m_{1}+m_{3}+u_{2}\right) \\
m_{2}
\end{array}\right]_{q}\left[\begin{array}{c}
\frac{1}{2}\left(m_{2}+u_{3}\right) \\
m_{3}
\end{array}\right]_{q},
$$

where $(q)_{0}=1,(q)_{m}=\prod_{a=1}^{m}\left(1-q^{a}\right)$, the $q$-binomial coefficient is defined for integer $m, n$ as

$$
\left[\begin{array}{c}
n \\
m
\end{array}\right]_{q}=\left\{\begin{array}{cl}
\frac{(q)_{n}}{(q)_{n-m}(q)_{m}} & \text { if } n \geq m \geq 0 \\
0 & \text { otherwise }
\end{array}\right.
$$




\begin{tabular}{|cclllrrc|}
\hline & $\Delta$ & $m_{1}$ & $m_{2}$ & $m_{3}$ & $u_{2}$ & $u_{3}$ & $L(\mathbf{m})$ \\
\hline$(1)$ & 0 & $\mathrm{e}$ & $\mathrm{e}$ & $\mathrm{e}$ & 0 & 0 & 0 \\
\hline$(2)$ & $\frac{2}{5}$ & $\mathrm{o}$ & $\mathrm{e}$ & $\mathrm{e}$ & 1 & 0 & 1 \\
$(3)$ & & $\mathrm{o}$ & $\mathrm{o}$ & $\mathrm{o}$ & 0 & 1 & 1 \\
\hline$(4)$ & $\frac{7}{5}$ & $\mathrm{e}$ & $\mathrm{e}$ & $\mathrm{o}$ & 1 & 0 & 3 \\
$(5)$ & & $\mathrm{e}$ & $\mathrm{o}$ & $\mathrm{e}$ & 0 & 1 & 3 \\
\hline$(6)$ & 3 & $\mathrm{o}$ & $\mathrm{e}$ & $\mathrm{o}$ & 0 & 0 & 6 \\
\hline$(7)$ & $\frac{1}{15}$ & $\mathrm{o}$ & $\mathrm{e}$ & $\mathrm{o}$ & 2 & 0 & $m_{2}$ \\
$(8)$ & & $\mathrm{e}$ & $\mathrm{e}$ & $\mathrm{e}$ & 2 & 0 & $m_{2}$ \\
$(9)$ & & $\mathrm{e}$ & $\mathrm{o}$ & $\mathrm{o}$ & 1 & 1 & $m_{2}$ \\
$(10)$ & & $\mathrm{o}$ & $\mathrm{o}$ & $\mathrm{e}$ & 1 & 1 & $m_{2}$ \\
$(11)$ & & $\{\mathrm{e}$ & $\mathrm{o}$ & $\mathrm{o}$ & & & \\
& & $+\mathrm{o}$ & $\mathrm{o}$ & $\mathrm{e}\}$ & 1 & -1 & $m_{1}-m_{3}$ \\
\hline$(12)$ & $\frac{2}{3}$ & $\mathrm{e}$ & $\mathrm{e}$ & $\mathrm{o}$ & 1 & 0 & $m_{2}+1$ \\
$(13)$ & & $\mathrm{o}$ & $\mathrm{e}$ & $\mathrm{e}$ & 1 & 0 & $m_{2}+1$ \\
$(14)$ & & $\{\mathrm{e}$ & $\mathrm{o}$ & $\mathrm{e}$ & & & \\
& & $+\mathrm{o}$ & $\mathrm{o}$ & $\mathrm{o}\}$ & 0 & -1 & $m_{1}-m_{3}+1$ \\
\hline
\end{tabular}

Table 1: Restrictions and linear translation terms for the characters $\widehat{\chi}_{\Delta}$ in equation (1.2). Here $\mathrm{e} \equiv$ even and $\mathrm{o} \equiv$ odd. Note that the characters $\widehat{\chi}_{1 / 15}$ and $\hat{\chi}_{2 / 3}$ have a two-term expression as well as one-term expressions.

$\mathbf{m}=\left(m_{1}, m_{2}, m_{3}\right)$, and $C_{A_{3}}$ is the Cartan matrix of the Lie algebra $A_{3}$ :

$$
C_{A_{3}}=\left(\begin{array}{rrr}
2 & -1 & 0 \\
-1 & 2 & -1 \\
0 & -1 & 2
\end{array}\right) .
$$

The restrictions on the integers $m_{a}$ in equation (1.2) depend on the character in question, and are such that $m_{a}$ are either even (e) or odd (o). These restrictions are listed, together with the $u_{a}$ and the linear translation terms $L(\mathbf{m})$, in table $\mathbb{1}$. We note that for characters other than $\hat{\chi}_{0}$ and $\widehat{\chi}_{3}$ there is more than one representation of the form (1.2), and that the formulas corresponding to lines $(1)-(7),(9),(12)-(13)$ in the table are special cases of the fermionic sum representations for Virasoro characters presented already in [18].

The modular invariant partition function of the conformal field theory associated with the three-state Potts model is written in a factorized form in terms of these characters [17]:

$$
\begin{aligned}
& (q \bar{q})^{-\frac{1}{30}} \widehat{Z}=\left[\chi_{0}(q)+\chi_{3}(q)\right]\left[\chi_{0}(\bar{q})+\chi_{3}(\bar{q})\right]+\left[\chi_{\frac{2}{5}}(q)+\chi_{\frac{7}{5}}(q)\right]\left[\chi_{\frac{2}{5}}(\bar{q})+\chi_{\frac{7}{5}}(\bar{q})\right] \\
& +2 \chi_{\frac{1}{15}}(q) \chi_{\frac{1}{15}}(\bar{q})+2 \chi_{\frac{2}{3}}(q) \chi_{\frac{2}{3}}(\bar{q}) .
\end{aligned}
$$


Here the variable $q(=\bar{q})$ is associated with contributions coming from right- (left-) moving excitations, as discussed in sect. 2 .

In this paper we construct a direct connection between the low-lying spectrum of the spin chain hamiltonian and the conformal field theory. We do so by computing the partition function of the spin chain in an appropriate scaling limit (see (1.12) below), obtaining expressions of the form (1.2) for the Virasoro characters. Our starting point is the quasi-particle nature of the spectrum.

A many-body system is said to have a quasi-particle spectrum if in the infinite-size limit the energy $E$ and momentum $P$ of the low-lying excitations above the ground state are of the form

$$
E-E_{G S}=\sum_{\alpha, \text { rules }} \sum_{j=1}^{m_{\alpha}} e_{\alpha}\left(P_{j}^{\alpha}\right), \quad P-P_{G S} \equiv \sum_{\alpha, \text { rules }} \sum_{j=1}^{m_{\alpha}} P_{j}^{\alpha}(\bmod 2 \pi),
$$

where $m_{\alpha}$ is the number of excitations of type $\alpha$ in a given state. The rules of composition in (1.6) depend on the model in question, and commonly include a fermionic exclusion rule

$$
P_{j}^{\alpha} \neq P_{k}^{\alpha} \quad \text { if } \quad j \neq k
$$

in which case the spectrum is said to be fermionic.

There are many cases where the excitation spectrum is gapless, i.e. one or more of the $e_{\alpha}\left(P^{\alpha}\right)$ vanish at some value of the momentum, say at $P^{\alpha}=0$, and

$$
e_{\alpha}\left(P^{\alpha}\right) \sim v_{\alpha}\left|P^{\alpha}\right| \quad \text { for } \quad P^{\alpha} \sim 0
$$

where $v_{\alpha}>0$ is the fermi velocity of the excitation of type $\alpha$.

The partition function of the quantum spin chain at temperature $T$ is the sum over all states,

$$
Z=\sum_{\{\text {states }\}} \mathrm{e}^{-E / k_{B} T}=\mathrm{e}^{-E_{G S} / k_{B} T} \sum_{\{\text {states }\}} \mathrm{e}^{-\left(E-E_{G S}\right) / k_{B} T},
$$

and the specific heat in the thermodynamic limit is defined by

$$
C(T)=-T \frac{\partial^{2} f}{\partial T^{2}}, \quad \text { where } \quad f=-k_{B} T \lim _{M \rightarrow \infty} \frac{1}{M} \ln Z .
$$


Here $M$ is the size of the system and the temperature $T$ has some fixed positive value. When the spectrum is of the form (1.8), at low temperature the specific heat is dominated by quasiparticle states (1.6) with vanishing single-particle energies and exhibits a linear $T$ behavior. Therefore, in order to extract this behavior it is necessary to consider only excitations of this type in the sum over states (1.9). We refer to the resulting partition function, in the limit $M \rightarrow \infty$ and with the ground state energy factored out, as the conformal partition function. More explicitly, the conformal field theory partition function (1.5) is obtained from

$$
\widehat{Z}=\lim \mathrm{e}^{E_{G S} / k_{B} T} Z
$$

in the limit

$$
T \rightarrow 0 \quad \text { and } \quad M \rightarrow \infty, \quad \text { with } \quad M T \text { fixed }
$$

Using (1.6) and (1.8) we see that $\widehat{Z}$ is a function of the variable

$$
q \equiv \exp \left(-\frac{2 \pi v}{M k_{B} T}\right)
$$

If there are no additional length scales in the problem, the $q \rightarrow 1$ behavior of $\widehat{Z}$ and the $T \rightarrow 0$ limit of the partition function in the thermodynamic limit (1.10) should match. Indeed, the leading $q \rightarrow 1$ behavior of $\widehat{Z}$ was computed in 18 from the expression for the characters (1.2), where it was shown that the linear behavior of the specific heat obtained in this way is the same as that obtained in the thermodynamic limit at low temperature [7, 19].

We also remark that the connection between these two different computations goes beyond giving just the same final result for the value of the specific heat coefficient. In the analysis of the $q \rightarrow 1$ behavior of sums generalizing (1.2) for characters of a large class of conformal field theories, one encounters [18 the same equations (involving dilogarithms) which appear in thermodynamic Bethe Ansatz analyses of the corresponding spin chains, as well as of factorizable scattering theories that are associated with certain integrable perturbations of the conformal field theory in question. We will say more about the relation between fermionic character sums and integrable perturbations in sect. 5 . 
In [4] it was shown for the anti-ferromagnetic three-state Potts chain that the sum over low-lying excitations with a massless dispersion relation (1.8) gives rise to the $D_{4}$ [20] modularinvariant partition functions of the $\mathbf{Z}_{4}$-parafermionic conformal field theory. In that model there are three different excitations, all having the same linear dispersion relation. In contrast, the spectrum of the ferromagnetic three-state Potts chain has a different structure. While there is only one type of quasi-particle excitation of the kind found for the anti-ferromagnetic case [5], there are two more excitations, which do not contribute to the energies at order one $\left(=M^{0}\right)$ but rather determine the degeneracy of states of the order one excitation spectrum [21], thus affecting the thermodynamics through entropy considerations. In the calculation of the partition function, where we take the energy of all excitations to have a linear dispersion relation, this can be viewed as a statement that the momentum range of these latter two excitations is microscopic (of order $M^{-1}$ ), instead of being macroscopic (order $M^{0}$ ) as it is for the quasi-particle excitation.

In sect. 2 we define the model and introduce the relevant Bethe equations, as well as the order one spectrum. In sect. 3 we use the finite-size studies of [21, 22] to extend the order one analysis of the spectrum [5] to order $1 / M$, and study the sectors of the partition function which give rise to the representations (1), (2), (4) and (6) in table 1 for the characters $\widehat{\chi}_{0}, \widehat{\chi}_{3}, \widehat{\chi}_{2 / 5}$ and $\hat{\chi}_{7 / 5}$. The sector of the partition function which corresponds to the character $\hat{\chi}_{1 / 15}$ is analyzed in sect. 4 . This gives a representation for $\widehat{\chi}_{1 / 15}$ in terms of five sums of the form (1.2). In sect. 5 we contrast the form (1.2) with the result of [13], and discuss the relation of these different fermionic representations for the conformal field theory characters to certain integrable massive extensions. 


\section{The gapless three-state Potts chain}

The gapless three-state Potts quantum spin chain of $M$ sites with periodic boundary conditions is defined by the hamiltonian

$$
H= \pm \frac{2}{\sqrt{3}} \sum_{j=1}^{M}\left\{X_{j}+X_{j}^{\dagger}+Z_{j} Z_{j+1}^{\dagger}+Z_{j}^{\dagger} Z_{j+1}\right\}
$$

where $Z_{M+1}=Z_{1}$ and for $j=1, \ldots, M$ the matrices $X_{j}$ and $Z_{j}$ are written as a direct product of $M 3 \times 3$ matrices:

$$
X_{j}=I \otimes I \otimes \cdots \otimes \underbrace{X}_{j^{t h}} \otimes \cdots \otimes I, \quad Z_{j}=I \otimes I \otimes \cdots \otimes \underbrace{Z}_{j^{t h}} \otimes \cdots \otimes I .
$$

Here $I$ is the identity matrix and

$$
X=\left(\begin{array}{ccc}
0 & 0 & 1 \\
1 & 0 & 0 \\
0 & 1 & 0
\end{array}\right), \quad Z=\left(\begin{array}{ccc}
1 & 0 & 0 \\
0 & \omega & 0 \\
0 & 0 & \omega^{2}
\end{array}\right), \quad \omega=\mathrm{e}^{2 \pi i / 3}
$$

The hamiltonian with the $(+)-\operatorname{sign}$ is referred to as the (anti-) ferromagnetic spin chain. The hamiltonian has a $\mathbf{Z}_{3}$ spin-rotation invariance and thus the $\mathbf{Z}_{3}$ charges $Q=0, \pm 1$ are good quantum numbers. In addition (2.1) is invariant under complex conjugation and hence the sectors $Q= \pm 1$ have equal eigenvalues and in $Q=0$ the eigenvalue $C= \pm 1$ of the charge conjugation operator is a good quantum number.

The hamiltonian (2.1) is derived from the two-dimensional critical three-state Potts model of classical statistical mechanics. The eigenvalues of the transfer matrix of the latter model satisfy functional equations [11], which, when specialized to the hamiltonian point [21], yield equations for the eigenvalues of the hamiltonian. These eigenvalues are given by

$$
E=\sum_{j=1}^{L} \cot \left(i \lambda_{j}+\frac{\pi}{12}\right)-\frac{2 M}{\sqrt{3}}, \quad L=2(M-|Q|), \quad Q=0, \pm 1
$$

where the rapidities $\lambda_{j}$ satisfy a set of equations of the form of Bethe equations:

$$
\left[\frac{\sinh \left(i \pi / 12-\lambda_{j}\right)}{\sinh \left(i \pi / 12+\lambda_{j}\right)}\right]^{2 M}=(-1)^{M+1} \prod_{k=1}^{L} \frac{\sinh \left(i \pi / 3-\left(\lambda_{j}-\lambda_{k}\right)\right)}{\sinh \left(i \pi / 3+\left(\lambda_{j}-\lambda_{k}\right)\right)}, \quad j=1, \ldots, L .
$$


The corresponding momentum, which is defined as the eigenvalue of the translation operator, is given by

$$
\mathrm{e}^{i P}=\prod_{k=1}^{L} \frac{\sinh \left(\lambda_{k}-i \pi / 12\right)}{\sinh \left(\lambda_{k}+i \pi / 12\right)} .
$$

The solutions of the Bethe equations are sets of (possibly complex) roots $\left\{\lambda_{j}\right\}$, and in the large lattice limit each root belongs to one of five different classes [21, the roots in each class having a fixed value of the imaginary part of $\lambda_{j}$ ㄹ:

$$
\lambda_{j} \text { is called }\left\{\begin{array}{l}
\lambda_{j}^{+} \\
\lambda_{j}^{-} \\
\lambda_{j}^{2 s} \\
\lambda_{j}^{-2 s} \\
\lambda_{j}^{n s}
\end{array}\right\} \quad \text { if } \quad \Im \mathrm{m}\left(\lambda_{j}\right)=\left\{\begin{array}{c}
0 \\
\pi / 2 \\
\pm \pi / 6 \\
\pm \pi / 3 \\
\pm \pi / 4
\end{array}\right\} .
$$

The last three classes of roots occur in complex conjugate pairs, and are referred to as complex pairs. We define $m_{\alpha}$ (where $\left.\alpha=+,-, 2 s,-2 s, n s\right)$ to be the number of roots in each class, complex pairs being counted once. A detailed analysis of the equations (2.5) was performed in [21]. We summarize those results of that paper which we will use here in the appendix.

The order one excitation spectrum obtained from (2.4)-(2.5) in the limit $M \rightarrow \infty$ was found in [5]. It was shown there that for the ferromagnetic case, the order one energy gaps can all be written in the quasi-particle form

$$
E-E_{G S}=\sum_{j=1}^{m_{+}} e_{+}\left(P_{j}^{+}\right),
$$

where $m_{+}=2 m_{n s}+3 m_{-}+4 m_{-2 s}$ and the single-particle energy is

$$
e_{+}\left(P_{j}^{+}\right)=6 \sin \left(\left|P_{j}^{+}\right| / 2\right) \quad 0 \leq P_{j}^{+} \leq 2 \pi
$$

so that the fermi velocity is $v=3$. The momentum of a single-particle state is expressed in terms of its rapidity $\lambda^{+}$as

$$
P\left(\lambda^{+}\right) \equiv \pi+4 \tan ^{-1}\left(\tanh 3 \lambda^{+}\right) \quad(\bmod 2 \pi)
$$

\footnotetext{
${ }^{2}$ Note that the definition of $\lambda$ here has a factor of $-1 / 2$ relative to the definition in [21].
} 
The number of states characterized by the same set $\left\{P_{j}^{+}\right\}$(and thus by the corresponding set of single-particle energies $\left.\left\{e_{+}\left(P_{j}^{+}\right)\right\}\right)$is, in the sector $Q=0$, 21]

$$
\left(\begin{array}{c}
m_{-}+m_{-2 s} \\
m_{-}
\end{array}\right)\left(\begin{array}{c}
2 m_{-}+2 m_{-2 s}+m_{n s} \\
m_{n s}
\end{array}\right) \text {, }
$$

where $\left(\begin{array}{l}a \\ b\end{array}\right)$ is the binomial coefficient and $m_{+}=2 m_{n s}+3 m_{-}+4 m_{-2 s}$. This stems from the fact that the other excitations $(n s,-2 s)$ carry no energy, yet states differing only in their content of $\left\{\lambda_{j}^{\alpha}\right\}_{\alpha=n s,-2 s}$ have to be counted individually.

In order to construct the scaled partition function (1.11) of the model, we extend the order one spectrum to momenta near zero. At such momenta, the energy is

$$
e_{+}\left(P^{+}\right)=\left\{\begin{array}{lll}
v P^{+} & \text {for } & P^{+} \sim 0 \\
v\left(2 \pi-P^{+}\right) & \text {for } & P^{+} \sim 2 \pi .
\end{array}\right.
$$

Note that there are no absolute value signs, and the momentum is no longer defined mod $2 \pi$. This amounts to extending the order one result (2.9) to order $1 / M$; however, at this order we must consider two additional contributions to the energy:

1. To order one, the excitations $n s$ and $-2 s$ contribute zero energy. However to order $1 / M$ they may carry energy, and indeed we find that $e_{\alpha}\left(P^{\alpha}\right)=v P^{\alpha}$ for $\alpha=n s,-2 s$, but with $P^{\alpha}$ restricted to only a microscopic range, of order $1 / M$. Here $v$ is the same as in equation (2.12).

2. Constant (independent of momentum or the number of excitations) contributions of order $1 / M$ to the energy must be accounted for. These contributions, which give the conformal dimensions $\Delta_{r, s}$, have been computed from functional equations for the transfer matrix by Klümper and Pearce [22].

From equation (2.6) and equations (A.18), (A.23) and (A.26) of the appendix, we see that the total momentum of any state can be written as

$$
P=\frac{2 \pi}{M}\left\{\sum_{j=1}^{m_{+}} \bar{I}_{j}^{+}+\sum_{j=1}^{m_{-2 s}} I_{j}^{-2 s}+\sum_{j=1}^{m_{n s}} I_{j}^{n s}+L\left(m_{\alpha}\right)\right\},
$$


where $L\left(m_{\alpha}\right)$ is some linear shift which depends on the sector under consideration. In equation (2.12) the energy depends linearly on the $P_{j}^{\alpha}$, which are quantized in units of $2 \pi / M$ and are directly related to the (half-) integers of the logarithmic Bethe equations (A.1) as:

$$
P_{j}^{+}=\frac{2 \pi}{M} I_{j}^{+}+\pi \equiv \frac{2 \pi}{M} \bar{I}_{j}^{+}, \quad P_{j}^{-2 s}=\frac{2 \pi}{M} I_{j}^{-2 s}, \quad P_{j}^{n s}=\frac{2 \pi}{M} I_{j}^{n s} .
$$

The energy can thus be expressed in terms of the $I_{j}^{\alpha}$.

The spectrum (and so the partition function) splits into different sectors of definite $\mathbf{Z}_{3}$ charge $Q=0, \pm 1$, and furthermore the sector $Q=0$ splits into subsectors of parity number $C= \pm 1$, corresponding to $m_{-}$being even $(C=1)$ or odd $(C=-1)$. Hence we can discuss separately each sector, which give rise to different characters, as in the anti-ferromagnetic case 41 .

\section{The characters in the sector $Q=0$}

The (half-) integers in this sector are chosen from the ranges (A.8)-(A.9), and hence we see from (2.14) that the $P_{j}^{\alpha}$ are chosen from the ranges of spacing $2 \pi / M$ with the following limits:

$$
\begin{gathered}
-\frac{2 \pi}{M}\left[\frac{1}{2}\left(m_{-}+m_{-2 s}-1\right)\right] \leq P_{j}^{+} \leq 2 \pi+\frac{2 \pi}{M}\left[\frac{1}{2}\left(m_{-}+m_{-2 s}-1\right)\right] \\
-\frac{2 \pi}{M}\left[\frac{1}{2}\left(m_{-}+m_{-2 s}-1\right)\right] \leq P_{j}^{-2 s} \leq \frac{2 \pi}{M}\left[\frac{1}{2}\left(m_{-}+m_{-2 s}-1\right)\right] \\
-\frac{2 \pi}{M}\left[\frac{1}{2}\left(2 m_{-}+2 m_{-2 s}+m_{n s}-1\right)\right] \leq P_{j}^{n s} \leq \frac{2 \pi}{M}\left[\frac{1}{2}\left(2 m_{-}+2 m_{-2 s}+m_{n s}-1\right)\right] .
\end{gathered}
$$

As is the case for the excitations of the anti-ferromagnetic chain [4], the range of single-particle momenta for the ' + '-excitations is macroscopic: it is of order $2 \pi$ for any finite $m_{\alpha}$ in the limit $M \rightarrow \infty$. In contrast, the ranges for $P_{j}^{n s}$ and $P_{j}^{-2 s}$ are of order $1 / M$ and allow only a finite number of states in the limit $M \rightarrow \infty$, for given $m_{\alpha}$. We refer to excitations with such microscopic momentum ranges as "ghost" excitations.

One expects that the partition function factorizes into right- and left-moving contributions, as in the anti-ferromagnetic case, so that the characters of the model are obtained by considering these contributions separately. However, in the ferromagnetic case only the $P_{j}^{+}$can be considered to be right- or left-moving, where right- (left-) movers indicates $P_{j}^{+} \sim 0\left(P_{j}^{+} \sim 2 \pi\right)$. 
When taking the limit $M \rightarrow \infty$, right- (left-) movers can be considered to lie on a semiinfinite range, since the range for $\mathrm{P}^{+}$is macroscopic, allowing for an infinite number of momentum states. Therefore, we rewrite the momentum range for right-movers in this limit as

$$
-\frac{2 \pi}{M}\left[\frac{1}{2}\left(m_{-}+m_{-2 s}-1\right)\right] \leq P_{j}^{+}<\infty \quad \text { for right-movers }
$$

replacing equation (3.1) above. For the left-movers it is convenient to replace $P^{+}$by $P^{+}-2 \pi$, so that the momentum range in the $M \rightarrow \infty$ limit is

$$
-\infty<P_{j}^{+} \leq \frac{2 \pi}{M}\left[\frac{1}{2}\left(m_{-}+m_{-2 s}-1\right)\right] \quad \text { for left-movers }
$$

and the dispersion relation (2.12) now reads

$$
e_{+}\left(P^{+}\right)= \begin{cases}v P^{+} & \text {for right-movers } \\ -v P^{+} & \text {for left-movers }\end{cases}
$$

There are four characters corresponding to the $Q=0$ sector (since there is a symmetry between right- and left-movers, below we restrict our attention to the right-movers):

1. The vacuum character $\widehat{\chi}_{0}$, which corresponds to the sector of the partition function with only right-movers and positive parity, $C=+1$;

2. The character $\widehat{\chi}_{3}$, which corresponds to the sector with only right-movers and negative parity, $C=-1$

3. The character $\widehat{\chi}_{2 / 5}$, which has one left-mover and the rest right-movers, with $C=+1$, and

4. $\widehat{\chi}_{7 / 5}$, which has only one left-mover and $C=-1$.

We will discuss in detail the construction of the partition function in the sector corresponding to $\widehat{\chi}_{0}$, item 1 above, and then outline the computation of the other characters. 


\subsection{Construction of the character $\hat{\chi}_{0}$}

The sector of the partition function (1.9) which has only right-movers and $Q=0, C=+1$ is computed as follows. The excitation energy is simply the sum over the individual excitations near $P^{\alpha} \sim 0$

$$
E-E_{G S}=v\left\{\sum_{j=1}^{m_{+}} P_{j}^{+}+\sum_{j=1}^{m_{-} 2 s} P_{j}^{-2 s}+\sum_{j=1}^{m_{n s}} P_{j}^{n s}\right\} .
$$

The partition function is the sum over all right-moving excitations with momentum ranges (3.2) - (3.4), subject to the fermionic exclusion rule (1.7), and the restriction that $m_{-}$be even. In table 2 we show the lowest energy states of in this sector. The general expression for the partition function in this sector is

$$
\widehat{Z}_{0}=\sum_{\{\text {states }\}} \mathrm{e}^{-v P / k_{B} T}=\sum_{\left\{I_{j}^{\alpha}\right\}} q\left(\sum_{j} \bar{I}_{j}^{+}+\sum_{j} I_{j}^{-2 s}+\sum_{j} I_{j}^{n s}\right),
$$

with $q$ defined as in equation (1.13). Here the $I_{j}^{\alpha}$ are restricted as in equation (A.8) in the appendix with $m_{-}$even. As in (3.4), in the limit $M \rightarrow \infty$ we have for right-moving '+'excitations

$$
-\frac{1}{2}\left(m_{-}+m_{-2 s}-1\right) \leq \bar{I}_{j}^{+}<\infty
$$

The restrictions on the integers are implemented by using two integer partitions, $Q_{m}(N ; n)$ and $Q_{m}(N) \equiv Q_{m}(N ; \infty)$, where $Q_{m}(N ; n)$ is the number of partitions of $N \geq 0$ into $m$ distinct non-negative integers each less than or equal to $n$. The partition function (3.8) subject to the restrictions (3.4), (3.2), (3.3) then becomes:

$$
\begin{aligned}
\widehat{Z}_{0}= & \sum_{\begin{array}{c}
m_{-}, m_{-2 s}, m_{n s}=0 \\
m_{-} \text {even } \\
m_{+}=2 m_{n s}+3 m_{-}+4 m_{-2 s}
\end{array}}^{\infty} \sum_{N_{+}, N_{-2 s}, N_{n s}=0}^{\infty} Q_{m_{+}}\left(N_{+}\right) q^{N_{+}-\frac{1}{2} m_{+}\left(m_{-2 s}+m_{-}-1\right)} \\
& \times Q_{m_{-2 s}}\left(N_{-2 s} ; m_{-2 s}+m_{-}-1\right) q^{N_{-2 s}-\frac{1}{2} m_{-2 s}\left(m_{-2 s}+m_{-}-1\right)} \\
& \times Q_{m_{n s}}\left(N_{n s} ; m_{n s}+2 m_{-2 s}+2 m_{-}-1\right) q^{N_{n s}-\frac{1}{2} m_{n s}\left(m_{n s}+2 m_{-2 s}+2 m_{-}-1\right)} .
\end{aligned}
$$

The exponents of $q$ above are essentially the total momenta of each type of excitation, i.e. the sums over the integers $N_{\alpha}=\sum_{j} I_{j}^{\alpha}$. The partitions count the number of times $q^{\sum_{\alpha} N_{\alpha}}$ 
occurs in the partition function, which is the number of ways $N_{\alpha}$ can be divided between $m_{\alpha}$ fermionic excitations.

The sum (3.10) can be re-expressed using the identity [23, 24]

$$
\sum_{N=0}^{\infty} Q_{m}(N ; n) q^{N}=q^{m(m-1) / 2}\left[\begin{array}{c}
n+1 \\
m
\end{array}\right]_{q},
$$

which, when $n \rightarrow \infty$, reduces to

$$
\sum_{N=0}^{\infty} Q_{m}(N) q^{N}=\frac{q^{m(m-1) / 2}}{(q)_{m}}
$$

Using these identities, (3.10) becomes

$$
\begin{array}{r}
\widehat{Z}_{0}=\sum_{\substack{m_{-}, m_{-2 s}, m_{n s}=0 \\
m_{+}=2 m_{n s+3}+3 m_{-}+4 m_{-2 s} \\
m_{-} \text {even }}}^{\infty} q^{-\frac{1}{2}\left(m_{+}+m_{-2 s}\right)\left(m_{-2 s}+m_{-}-1\right)-\frac{1}{2} m_{n s}\left(m_{n s}+2 m_{-2 s}+2 m_{-}-1\right)} \\
\times q^{\frac{1}{2} m_{+}\left(m_{+}-1\right)+\frac{1}{2} m_{-2 s}\left(m_{-2 s}-1\right)+\frac{1}{2} m_{n s}\left(m_{n s}-1\right)} \\
\times \frac{1}{(q)_{m_{+}}}\left[\begin{array}{c}
m_{-2 s}+m_{-} \\
m_{-2 s}
\end{array}\right]_{q}\left[\begin{array}{c}
m_{n s}+2 m_{-2 s}+2 m_{-} \\
m_{n s}
\end{array}\right]_{q} .
\end{array}
$$

The form of the sum may be further simplified by changing variables to

$$
m_{1}=m_{+}, \quad m_{2}=2 m_{-}+2 m_{-2 s}, \quad m_{3}=m_{-},
$$

which results in the expression (1.2) with the restriction that all $m_{a}$ are even and $L(\mathbf{m})=$ $0, u_{a}=0$. This is the expression listed on line (1) of table 1.

This expression is quite different in form from the one given in (1.1). Nevertheless we find that

$$
\widehat{Z}_{0}=\widehat{\chi}_{0}
$$

This has been verified as an equality between the series expansions of the two expressions to order $q^{200}$, using Mathematica.

\subsection{Construction of $\hat{\chi}_{3}$}

In table 3 we present the lowest energy states of the sector $C=-1$, where all $m_{+}$are right-

movers. The calculation of the partition function $\widehat{Z}_{3}$ is identical to that of the last section, 
except that now $m_{-}$is odd in equations (3.10) and (3.13). Using series expansions, we verify that the resulting expression $\widehat{Z}_{3}$ is equal to $q^{3} \widehat{\chi}_{3}$ to order $q^{200}$. With the change of variables (3.14), this results in the expression on line (6) of table 11 .

\subsection{Construction of $\hat{\chi}_{2 / 5}$ and $\hat{\chi}_{7 / 5}$}

The characters $\hat{\chi}_{2 / 5}$ and $\hat{\chi}_{7 / 5}$ occur when one of the $m_{+}$is a left-mover, and all the rest are right-movers. This amounts to setting $m_{+}=2 m_{n s}+3 m_{-}+4 m_{-2 s}-1$ in the partition sum (3.10) and (3.13). Also, there is an additive term to the momentum of the form $\frac{\pi v}{M}\left(m_{-}+m_{n s}-1\right)$, which is the lowest energy state of the single left-moving '+'-excitation allowed by equation (3.5). The character $\widehat{\chi}_{2 / 5}$ occurs for $C=+1$, i.e. $m_{-}$even, and the character $\hat{\chi}_{7 / 5}$ occurs for $C=-1, m_{-}$odd. We tabulate the lowest energy states for these two sectors in tables $⿴$ and 5. The expression for the resulting partition functions is

$$
\begin{aligned}
\widehat{Z}_{2 / 5(7 / 5)}= & \sum_{\substack{m_{+}, m_{-2 s}, m_{n s}=0 \\
m_{+}=2 m_{n s}+3 m_{-}+4 m_{-2 s}-1 \\
m_{-} \text {even (odd) }}}^{\infty} \sum_{N_{\alpha}=0}^{\infty} q^{\frac{1}{2}\left(m_{-}+m_{-2 s}-1\right)} Q_{m_{+}}\left(N_{+}\right) q^{N_{+}-\frac{1}{2} m_{+}\left(m_{-2 s}+m_{-}-1\right)} \\
& \times Q_{m_{-2 s}}\left(N_{-2 s} ; m_{-2 s}+m_{-}-1\right) q^{N_{-2 s}-\frac{1}{2} m_{-2 s}\left(m_{-2 s}+m_{-}-1\right)} \\
& \times Q_{m_{n s}}\left(N_{n s} ; m_{n s}+2 m_{-2 s}+2 m_{-}-1\right) q^{N_{n s}-\frac{1}{2} m_{n s}\left(m_{n s}+2 m_{-2 s}+2 m_{-}-1\right)}
\end{aligned}
$$

Using the identities (3.11)-(3.12) and the change of variables (3.14), $\widehat{Z}_{2 / 5}$ and $q^{-1} \widehat{Z}_{7 / 5}$ are brought to the form (1.2) with the restrictions listed on lines (2) and (4) of table 1, i.e. we find that

$$
\widehat{Z}_{2 / 5}=\widehat{\chi}_{2 / 5}, \quad \widehat{Z}_{7 / 5}=q \widehat{\chi}_{7 / 5}
$$

The other expressions for the two characters $\widehat{\chi}_{2 / 5}$ and $\widehat{\chi}_{7 / 5}$, corresponding to lines (3) and (5) of that table, are conjectured forms. Using a power series expansion, all these forms were shown to be equal to the corresponding expressions (1.1) for $\widehat{\chi}_{2 / 5}$ and $\widehat{\chi}_{7 / 5}$, to order $q^{200}$. 


\section{The Sector $Q=1$}

The analysis of this sector is more involved than for the $Q=0$ sector, since (see appendix) there are five different sub-sectors to be considered, where the integers range over different intervals. Each of these sub-sectors gives rise to a separate sum in the sector of the partition function corresponding to right-moving excitations. The resulting five sums add together to form the Virasoro character $\widehat{\chi}_{1 / 15}$, as we now describe in more detail.

The momentum ranges are given by the integer ranges (A.8) and (A.10)-(A.14) in the appendix, and the relations (A.18), (A.23) and (A.26) of the total momentum to the integers. We will present the computation for each sub-sector separately, where for each sub-sector all '+'-excitations are right-movers.

1. $m_{-}-m_{++}=+1$ : Here we see from equation (A.6) that $m_{+}=2 m_{n s}+3 m_{-}+4 m_{-2 s}-2$, and the lowest energy states are shown in table 6, where the integer range is that of equation (A.10). The partition sum starts with $m_{-}=1$, since $m_{-}>m_{++} \geq 0$ :

$$
\begin{gathered}
\widehat{Z}_{1 / 15}^{(1)}=\sum_{m_{-}=1}^{\infty} \sum_{\substack{m_{n s}, m_{-2 s}=0 \\
m_{+}=2 m_{n s}+3 m_{-}+4 m_{-2 s}-2}}^{\infty} q^{\frac{1}{2} m_{+}\left(m_{+}-1\right)+\frac{1}{2} m_{+}\left(3-m_{-}-m_{-2 s}\right)} \frac{1}{(q)_{m_{+}}} \\
\times q^{\frac{1}{2} m_{-2 s}\left(m_{-2 s}-1\right)+\frac{1}{2} m_{-2 s}\left(3-m_{-}-m_{-2 s}\right)}\left[\begin{array}{c}
m_{-2 s}+m_{-}-2 \\
m_{-2 s}
\end{array}\right]_{q} \\
\times q^{\frac{1}{2} m_{n s}\left(m_{n s}-1\right)+\frac{1}{2} m_{n s}\left(3-2 m_{-}-2 m_{-2 s}-m_{n s}\right)}\left[\begin{array}{c}
m_{n s}+2 m_{-2 s}+2 m_{-}-2 \\
m_{n s}
\end{array}\right]_{q} .
\end{gathered}
$$

2. $m_{-}-m_{++}=-1$ : From the sum rule (A.6) we see that $m_{+}=2 m_{n s}+3 m_{-}+4 m_{-2 s}+2$, and the integer range is given by (A.11). The lowest energy states are shown in table $\square$, and the general expression for the partition function is:

$$
\begin{aligned}
\widehat{Z}_{1 / 15}^{(2)}= & \sum_{\substack{m_{-}, m_{n s, m_{-2 s}=0} \\
m_{+}=2 m_{n s}+3 m_{-}+4 m_{-2 s}+2}}^{\infty} q^{\frac{1}{2} m_{+}\left(m_{+}-1\right)+\frac{1}{2} m_{+}\left(1-m_{-}-m_{-2 s}\right)} \frac{1}{(q)_{m_{+}}} \\
& \times q^{\frac{1}{2} m_{-2 s}\left(m_{-2 s}-1\right)+\frac{1}{2} m_{-2 s}\left(1-m_{-}-m_{-2 s}\right)}\left[\begin{array}{c}
m_{-2 s}+m_{-} \\
m_{-2 s}
\end{array}\right]_{q}
\end{aligned}
$$




$$
\times q^{\frac{1}{2} m_{n s}\left(m_{n s}-1\right)+\frac{1}{2} m_{n s}\left(-1-2 m_{-}-2 m_{-2 s}-m_{n s}\right)}\left[\begin{array}{c}
m_{n s}+2 m_{-2 s}+2 m_{-}+2 \\
m_{n s}
\end{array}\right]_{q} .
$$

3. $m_{-}=m_{++}=0$ : Since $m_{-}=0$, there are no $-2 s$ excitations. The relevant momentum range is obtained from the integer range (A.12), and the lowest energy states are listed in table 8. The general expression for the partition function is a simple sum over $n s$ excitations, with $m_{+}=2 m_{n s}$ :

$$
\widehat{Z}_{1 / 15}^{(3)}=\sum_{\substack{m_{n s}=0 \\ m_{+}=2 m_{n s}}}^{\infty} \frac{q^{m_{+}\left(m_{+}+1\right) / 2}}{(q)_{m_{+}}}=\sum_{m_{n s}=0}^{\infty} \frac{q^{m_{n s}\left(2 m_{n s}+1\right)}}{(q)_{2 m_{n s}}} .
$$

4. $m_{-}=m_{++} \neq 0$ : There are two sub-sectors with this characteristic, corresponding to the integer ranges (A.13) and (A.14). These integer ranges are asymmetric, so there is a shift term in the total momentum, as shown in (A.23) and (A.26), of the form $\mp \frac{2 \pi v}{M}\left(\frac{1}{2} m_{n s}+m_{-}+m_{-2 s}\right)$. For both of these sectors $m_{+}=2 m_{n s}+3 m_{-}+4 m_{-2 s}$. The lowest energy states are listed in tables 9 and 10. The sums take the forms, for the integer range $(\mathrm{A} .13)$ :

$$
\begin{array}{r}
\widehat{Z}_{1 / 15}^{(4)}=\sum_{m_{-}=1}^{\infty} \sum_{\substack{m_{n s}, m_{-2 s}=0 \\
m_{+}=2 m_{n s}+3 m_{-}+4 m_{-2 s}}}^{\infty} q^{-\left(m_{-}+m_{-2 s}+\frac{1}{2} m_{n s}\right)} q^{\frac{1}{2} m_{+}\left(m_{+}-1\right)+\frac{1}{2} m_{+}\left(3-m_{-}-m_{-2 s}\right)} \frac{1}{(q)_{m_{+}}} \\
\times q^{\frac{1}{2} m_{-2 s}\left(m_{-2 s}-1\right)+\frac{1}{2} m_{-2 s}\left(1-m_{-}-m_{-2 s}\right)}\left[\begin{array}{c}
m_{-2 s}+m_{-} \\
m_{-2 s}
\end{array}\right]_{q} \\
\times q^{\frac{1}{2} m_{n s}\left(m_{n s}-1\right)+\frac{1}{2} m_{n s}\left(2 m_{-}-2 m_{-2 s}-m_{n s}\right)}\left[\begin{array}{c}
m_{n s}+2 m_{-2 s}+2 m_{-} \\
m_{n s}
\end{array}\right]_{q},
\end{array}
$$

and for the integer range (A.14):

$$
\begin{array}{r}
\widehat{Z}_{1 / 15}^{(5)}=\sum_{m_{-}=1}^{\infty} \sum_{\substack{m_{n s}, m_{-2 s}=0 \\
m_{+}=2 m_{n s}+3 m_{-}+4 m_{-2 s}}}^{\infty} q^{\left(m_{-}+m_{-2 s}+\frac{1}{2} m_{n s}\right)} q^{\frac{1}{2} m_{+}\left(m_{+}-1\right)+\frac{1}{2} m_{+}\left(1-m_{-}-m_{-2 s}\right)} \frac{1}{(q)_{m_{+}}} \\
\times q^{\frac{1}{2} m_{-2 s}\left(m_{-2 s}-1\right)+\frac{1}{2} m_{-2 s}\left(3-m_{-}-m_{-2 s}\right)}\left[\begin{array}{c}
m_{-2 s}+m_{-} \\
m_{-2 s}
\end{array}\right]_{q} \\
\times q^{\frac{1}{2} m_{n s}\left(m_{n s}-1\right)+\frac{1}{2} m_{n s}\left(2-2 m_{-}-2 m_{-2 s}-m_{n s}\right)}\left[\begin{array}{c}
m_{n s}+2 m_{-2 s}+2 m_{-} \\
m_{n s}
\end{array}\right]_{q} .
\end{array}
$$


Finally, we find that

$$
\sum_{a=1}^{5} \widehat{Z}_{1 / 15}^{(a)}=\widehat{\chi}_{1 / 15} .
$$

This is a five-sum expression for the character $\widehat{\chi}_{1 / 15}$, where each summand can be expressed in the form (1.2). In addition to this form, one can find the forms listed in table 1 for the character $\hat{\chi}_{1 / 15}$. Again, although all these forms are quite different from that of (1.1), they have been shown to be equal to order $q^{200}$.

It remains to consider the character $\hat{\chi}_{2 / 3}$. Here, however, no analysis corresponding to the above five-term sum form is availible. The conjectured forms on lines (12)-(14) of table 1 have been verified to order $q^{200}$.

\section{Discussion}

The forms of the expressions (1.1) and (1.2) for the characters of the ferromagnetic three-state Potts conformal field theory deserve to be called "different", even though the expressions are equal. The question thus arises as to what is meant by the word different, how many different forms there are, and what their significance is. We know of at least four different forms for the characters of the three-state Potts. One is the Rocha-Caridi form (1.1), the second is the form of Kac and Peterson [25] and Jimbo and Miwa [26], the third is that of Lepowsky and Primc [13], and the fourth is the form (1.2). Each of these forms is sufficiently different to warrant a separate discussion.

1. The expression (1.1) for the Virasoro characters, which are [27 branching functions of the coset $\frac{\left(A_{1}^{(1)}\right)_{3} \times\left(A_{1}^{(1)}\right)_{1}}{\left(A_{1}^{(1)}\right)_{4}}$, is what we refer to as a bosonic sum representation. This stems from the presence of the factor $(q)_{\infty}^{-1}$, which represents a bosonic partition function and can be understood in terms of the Feigin-Fuchs-Felder construction [28, 29] of the Virasoro minimal series [30] $\mathcal{M}\left(p, p^{\prime}\right)$ to which the three-state Potts conformal field theory belongs, being $\mathcal{M}(5,6)$ in this notation.

2. The second form is also a bosonic expression which can be obtained by viewing this 
conformal field theory as that of $\mathbf{Z}_{3}$-parafermions [16], where the characters of the corresponding $\mathbf{Z}_{3}$-parafermionic algebra are 20] the branching functions of the coset $\frac{\left(A_{1}^{(1)}\right)_{3}}{U(1)}$. Another description of the same conformal field theory is as a minimal model with respect to the $W_{3}$ algebra [31], where the corresponding coset construction is $\frac{\left(A_{2}^{(1)}\right)_{1} \times\left(A_{2}^{(1)}\right)_{1}}{\left(A_{2}^{(1)}\right)_{2}}$. The latter construction is related by level-rank duality [32] to $\frac{\left(A_{1}^{(1)}\right)_{3}}{U(1)}$, and the branching functions are in fact the same. They are given by the Hecke indefinite forms of [25, 26] (or alternative but very similar sum representations of [33])

$$
\begin{array}{r}
q^{\frac{1}{30}} b_{m}^{l}(q)=\frac{q^{h_{m}^{l}}}{(q)_{\infty}^{2}}\left[\left(\sum_{s \geq 0} \sum_{n \geq 0}-\sum_{s<0} \sum_{n<0}\right)(-1)^{s} q^{s(s+1) / 2+(l+1) n+(l+m) s / 2+5(n+s) n}\right. \\
\quad+\left(\sum_{s>0} \sum_{n \geq 0}-\sum_{s \leq 0} \sum_{n<0}\right)(-1)^{s} q^{s(s+1) / 2+(l+1) n+(l-m) s / 2+5(n+s) n]}
\end{array}
$$

where the $h_{m}^{l}$ are

$$
h_{m}^{l}=\frac{l(l+2)}{20}-\frac{m^{2}}{12} .
$$

Here $l=0,1,2, \quad l-m$ is even, and the formulas are valid for $|m| \leq l$ while for $|m|>l$ one uses the symmetries

$$
b_{m}^{l}=b_{-m}^{l}=b_{m+6}^{l}=b_{3-m}^{3-l} .
$$

The partition function (1.5) is expressed as a diagonal bilinear form in terms of the $b_{m}^{l}$, through

$$
\chi_{0}+\chi_{3}=b_{0}^{0}, \quad \chi_{2 / 5}+\chi_{7 / 5}=b_{0}^{2}, \quad \chi_{1 / 15}=b_{2}^{2}, \quad \chi_{2 / 3}=b_{2}^{0} .
$$

Note that two of the $b_{m}^{l}$ split into a sum of a pair of Virasoro characters, corresponding to a more refined splitting of the spectrum of the hamiltonian into various sectors. Also, the expressions (1.1) have only one factor of $(q)_{\infty}^{-1}$ while the ones in (5.1) have two. Thus whereas (1.1) can be said to be based on one boson, (5.1) is based on two bosons.

3. The third form is a fermionic sum representation for the branching functions $b_{m}^{l}$ which was obtained by Lepowsky and Primc [13]:

$$
q^{1 / 30} b_{2 Q-l}^{l}(q)=q^{\frac{l(2-l)}{16}} \sum_{\substack{m_{1}, m_{2}=0 \\ m_{1}-m_{2}=Q(\bmod 3)}}^{\infty} \frac{q^{\mathbf{m} C_{A_{2}}^{-1} \mathbf{m}^{t}+L_{l}(\mathbf{m})}}{(q)_{m_{1}}(q)_{m_{2}}}
$$


where $C_{A_{2}}=\left(\begin{array}{rr}2 & -1 \\ -1 & 2\end{array}\right)$ is the Cartan matrix of the Lie algebra $A_{2}$, and $L_{0}(\mathbf{m})=0$, $L_{1}(\mathbf{m})=\left(2 m_{1}+m_{2}\right) / 3, L_{2}(\mathbf{m})=\left(m_{1}+2 m_{2}\right) / 3$. This expression can be interpreted [34] in terms of two $C$-conjugate fermionic quasi-particles carrying $\mathbf{Z}_{3}$ charges \pm 1 , both having macroscopic momentum ranges. For example, for $l=0$ these ranges are

$$
\frac{2 \pi}{M}\left[\frac{1}{2}+\frac{1}{2}\left(\frac{m_{1}+2 m_{2}}{3}\right)\right] \leq P_{j}^{1}<\infty, \quad \frac{2 \pi}{M}\left[\frac{1}{2}+\frac{1}{2}\left(\frac{2 m_{1}+m_{2}}{3}\right)\right] \leq P_{j}^{2}<\infty,
$$

where the $P_{j}^{\alpha}\left(j=1, \ldots, m_{\alpha}\right)$ are chosen from a grid with spacing $2 \pi / M$.

4. The fourth form is the fermionic sum representation (1.2) which has one genuine quasiparticle with a macroscopic momentum range and two "ghost" quasi-particles, whose momenta are limited to a microscopic range, e.g. equations (3.1)-(3.3).

The existence of different fermionic sum representations for characters is closely related to the fact that one conformal field theory may have several integrable perturbations, characterized by the conformal dimensions $(\Delta, \bar{\Delta})$ of certain perturbing relevant operators. In 34 this observation was made in connection with the representations of the critical Ising characters as related to either (i) the coset $\frac{\left(A_{1}^{(1)}\right)_{1} \times\left(A_{1}^{(1)}\right)_{1}}{\left(A_{1}^{(1)}\right)_{2}}$ where the character formulas are written in terms of a single quasi-particle and the associated perturbation is by the $(1 / 2,1 / 2)$ operator, or (ii) the coset $\frac{\left(E_{8}^{(1)}\right)_{1} \times\left(E_{8}^{(1)}\right)_{1}}{\left(E_{8}^{(1)}\right)_{2}}$ which has eight quasi-particles and is associated with the $(1 / 16,1 / 16)$ perturbation [35]. In each case the perturbation can be thought of as giving masses to the fermionic quasi-particles.

A similar discussion can be given for the two different fermionic representations (5.5) and (1.2) of the critical three-state Potts model. Consider first (5.5), which was interpreted as having two fermionic quasi-particles of $\mathbf{Z}_{3}$ charge \pm 1 . This is to be compared with the $(2 / 5,2 / 5) S_{3}$-symmetric perturbation of the three-state Potts conformal field theory, which was argued in [36] to be integrable and to have a spectrum which consists of a $\mathbf{Z}_{3}$-doublet of massive particles, whose scattering is described by the factorizible $S$-matrix found in [37]. Here the perturbation can again be thought of as giving mass to the two fermionic quasi- 
particles. This perturbation is also to be compared with the massive $r=5$ RSOS model (or hard squares with diagonal interactions) [38], whose spectrum on the lattice [6] in regime II consists of two excitations with $\mathbf{Z}_{3}$ charge \pm 1 .

In the same spirit it is natural to associate the fermionic sum representation (1.2) with the $C$-even $(2 / 3,2 / 3)^{+}$perturbation. This subleading magnetic perturbation breaks the $S_{3}$ symmetry down to $\mathbf{Z}_{2}$. The related statistical mechanics system is the $N=3$ model of Kashiwara and Miwa [39 (also given as the $D_{4}$ model of Pasquier 40, obtained from the $r=6$ RSOS model of Andrews, Baxter and Forrester [41] by an orbifold construction [42]). In the notation of 40, 41] the lattice models are to be considered in the regimes III-IV. The perturbed conformal field theory is the $p=5$ case of the $\left(\Delta_{1,3}, \Delta_{1,3}\right)$-perturbed minimal models $\mathcal{M}(p, p+1)$, which have been discussed in [35] [43]-52] where it is seen that the sign of the coupling to the perturbing operator leads to qualitatively very different effects.

In the case of negative coupling constant (using the conventions of [35]) the perturbed theory becomes massive. This is to be compared to the massive regime III of the models of [39, 40, 41], where excitation energies have been computed in [53]. We interpret this direction of the perturabtion as giving mass to the quasi-particle $m_{1}$ of (1.2).

The more interesting case is the one where the coupling constant is positive. Now the integrable perturbed conformal field theory remains massless [35, 48], even though scale invariance is broken, and flows [49, 51, 52, 54] from the three-state Potts conformal field theory of central charge $4 / 5$ to the conformal field theory of the tricritical Ising model of central charge 7/10. This suggests an interpretation in terms of the representation (1.2), where we note that under the restriction to the sector where there are no "ghost" excitations of type $m_{3}$ the fermionic representations for the three-state Potts characters reduce to fermionic representations [18] for the characters of the tricritical Ising model. Specifically, restricting the summation in (1.2) by setting $m_{3}=0$, the formulas corresponding to lines (1), (2), (5), (8), (10) and (13) of table 1 reduce to expressions for the $c=7 / 10$ Virasoro characters $\widehat{\chi}_{\Delta}$ with $\Delta=0,7 / 16,3 / 2,3 / 80,1 / 10$ and $3 / 5$, respectively. The crucial point making this possible is the fact that (four times) the 
quadratic form in the fermionic sum representations of the $c=7 / 10$ characters is the Cartan matrix of $A_{2}$, which is precisely the minor, obtained by omitting the last row and column, of the quadratic form $C_{A_{3}}$ in ([1.2). More generally, we find from [18 that the fermionic form of the characters of the unitary minimal model $\mathcal{M}(p, p+1)$ with one quasi-particle and $p-3$ "ghosts" reduces to character formulas for $\mathcal{M}(p-1, p)$ when the last ghost is omitted, the corresponding massless flows being the ones discussed in 43, 44, 48].

Acknowledgements: We would like to thank Dr. G. Albertini and Prof. V.V. Bazhanov for fruitful discussions. The work of RK and BMM is partially supported by the NSF, grant DMR-9106648, and that of EM by the NSF grant 91-08054. 


\section{A Appendix: Logarithmic Bethe Equations}

We recall here some results [21] concerning the classification of the solutions of the Bethe equations corresponding to the eigenvalues of the hamiltonian (2.1).

Not all the roots $\lambda_{j}^{\alpha}$ (cf. equation (2.7)) in a given solution of the Bethe equations (2.5) are independent of one another, and in order to discuss the relations between them we introduce the logarithmic Bethe equations. By taking the logarithm of the Bethe equations (2.5), we can classify the sets $\left\{\lambda_{j}^{\alpha}\right\}$ more easily. Doing this introduces integers or half-integers associated with the choice of branch of the logarithm. The equations for the complex pairs are first multiplied together. After taking the logarithm, we obtain five sets of equations, one for each class of roots, referred to as the logarithmic Bethe equations:

$$
f_{\alpha} \frac{2 \pi}{M} I_{j}^{\alpha}=t_{\alpha}\left(\lambda_{j}^{\alpha}\right)-\frac{1}{M} \sum_{\beta= \pm, \pm 2 s, n s} \sum_{k=1}^{m_{\beta}} \Theta_{\alpha \beta}\left(\lambda_{j}^{\alpha}-\lambda_{k}^{\beta}\right), \quad \alpha \in\{+,-, 2 s,-2 s, n s\},
$$

where $f_{\alpha}=2$ for $\alpha=n s$ and is 1 otherwise $\}$, and where the functions $\Theta_{\alpha \beta}$ and $t_{\alpha}$ are defined as follows. Let $s_{\alpha}(\lambda) \equiv \sinh (i \alpha-\lambda) / \sinh (i \alpha+\lambda)$, then

$$
\begin{aligned}
& t_{\alpha}\left(\lambda_{j}^{\alpha}\right)= \begin{cases}-2 i \ln \left( \pm s_{\pi / 12}\left(\lambda_{j}^{ \pm}\right)\right) & \alpha= \pm \\
-2 i \ln \left(s_{\pi / 12}\left(\lambda_{j}^{\alpha}\right) s_{\pi / 12}\left(\lambda_{j}^{\alpha *}\right)\right) & \alpha= \pm 2 s, n s\end{cases}
\end{aligned}
$$

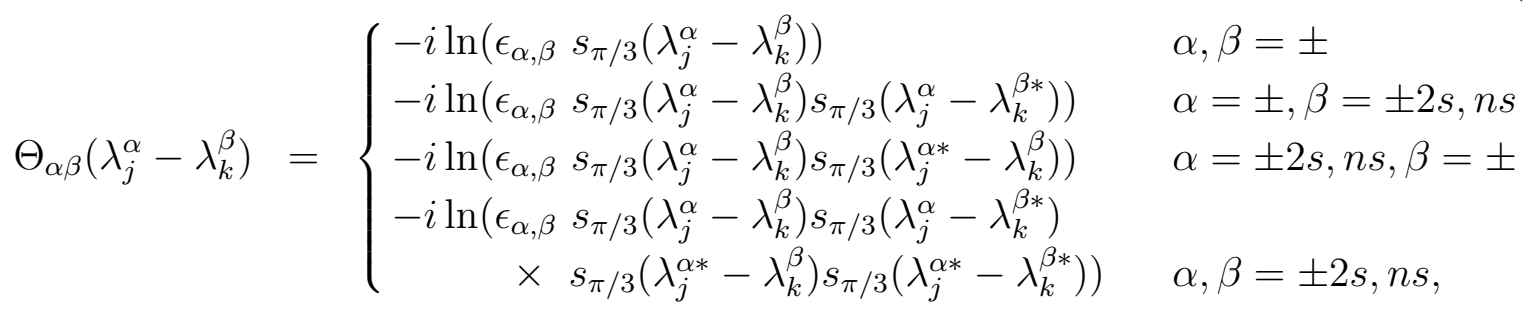

where the symmetric tensor $\epsilon_{\alpha, \beta}$ is defined by $\epsilon_{+,-}=\epsilon_{-, 2 s}=\epsilon_{+,-2 s}=\epsilon_{2 s, 2 s}=\epsilon_{-2 s,-2 s}=-1$ and the other $\epsilon_{\alpha, \beta}$ are 1. $\epsilon_{\alpha, \beta}$ is chosen so that $\Theta_{\alpha \beta}\left(\lambda^{\alpha}-\lambda^{\beta}\right)=0$ when $\Re \mathrm{e} \lambda^{\alpha}=\Re \mathrm{e} \lambda^{\beta}$. All logarithms in (A.2) and (A.3) are chosen such that $-\pi<\Im \mathrm{m} \ln z<\pi$. Each set of (half-) integers $\left\{I_{j}^{\alpha}\right\}$ uniquely specifies a set of roots $\left\{\lambda_{j}^{\alpha}\right\}$. Note that the sets contain either integers or half-integers, depending on $m_{\alpha}$.

\footnotetext{
${ }^{3}$ Note that the factor $f_{n s}$ was not present in the definitions of [21]. This amounts to a redefinition of the integers $I^{n s}$ discussed there.
} 
For the sector $Q=0$, there is a restriction on the number $m_{+}$of the form

$$
m_{+}=2 n_{n s}+3 m_{-}+4 m_{-2 s} .
$$

In addition, the total number of roots is $2 M$ (see equation (2.4)), so that

$$
M=m_{2 s}+2 m_{n s}+3 m_{-2 s}+2 m_{-} .
$$

For the sector $Q= \pm 1$, we define the number $m_{++}$which has the property that $m_{-}-m_{++}=$ $0, \pm 1$. For this sector we have the sum rule

$$
m_{+}=2 n_{n s}+m_{-}+2 m_{++}+4 m_{-2 s}
$$

and since the total number of roots is $2(M-1)$ (see equation $(2.4)$ ), we have

$$
M-1=m_{2 s}+2 m_{n s}+3 m_{-2 s}+m_{-}+m_{++} .
$$

The (half-) integers in equation (A.1) are not all independent, as the set $\left\{I_{j}^{2 s}\right\}$ and $\left\{I_{j}^{-}\right\}$ are completely determined from the sets $\left\{I_{j}^{+}\right\}$and $\left\{I_{j}^{-2 s}\right\}$, respectively. The ground state of the ferromagnetic chain consists of a sea of $2 s$-excitations, that is the integers $\left\{I_{j}^{2 s}\right\}$ fill a symmetric interval about zero, and all other sets of integers are null sets. Therefore, for convenience, we take the sets $\left\{I_{j}^{+}\right\},\left\{I_{j}^{-2 s}\right\}$ and $\left\{I_{j}^{n s}\right\}$ to be the independent sets in discussing the ferromagnetic case. Those (half-) integers are then freely chosen from the intervals

$$
\begin{aligned}
-\frac{1}{2}\left[M+m_{-}+m_{-2 s}-a_{\ell}^{(1)}\right] & \leq I_{j}^{+} \leq+\frac{1}{2}\left[M+m_{-}+m_{-2 s}-a_{r}^{(1)}\right] \\
-\frac{1}{2}\left[m_{-}+m_{-2 s}-a_{r}^{(1)}\right] & \leq I_{j}^{-2 s} \leq+\frac{1}{2}\left[m_{-}+m_{-2 s}-a_{\ell}^{(1)}\right] \\
-\frac{1}{2}\left[2 m_{-}+2 m_{-2 s}+m_{n s}-a_{\ell}^{(2)}\right] & \leq I_{j}^{n s} \leq+\frac{1}{2}\left[2 m_{-}+2 m_{-2 s}+m_{n s}-a_{r}^{(2)}\right]
\end{aligned}
$$

with a fermionic exclusion rule: $I_{j}^{\alpha} \neq I_{k}^{\alpha}$ for $j \neq k$. The numbers $a_{\ell}$ and $a_{r}$ depend on the sector in question. For the $Q=0$ sector,

$$
a_{\ell}^{(1)}=a_{r}^{(1)}=a_{\ell}^{(2)}=a_{r}^{(2)}=1 \quad \text { for } \quad Q=0
$$


In the $Q= \pm 1$ sectors, there are five separate sub-sectors to be considered, depending on the value of $m_{++}$introduced above:

$$
\begin{array}{lll}
\text { For } & m_{-}-m_{++}=+1: & a_{\ell}^{(1)}=a_{r}^{(1)}=3, a_{\ell}^{(2)}=a_{r}^{(2)}=3 \\
\text { For } & m_{-}-m_{++}=-1: & a_{\ell}^{(1)}=a_{r}^{(1)}=1, a_{\ell}^{(2)}=a_{r}^{(2)}=-1 \\
\text { For } & m_{-}=m_{++}=0: & a_{\ell}^{(1)}=a_{r}^{(1)}=2, a_{\ell}^{(2)}=a_{r}^{(2)}=1 \\
\text { For } & m_{-}=m_{++} \neq 0: & a_{\ell}^{(1)}=3, a_{r}^{(1)}=1, a_{\ell}^{(2)}=0, a_{r}^{(2)}=2 \\
\text { For } & m_{-}=m_{++} \neq 0: & a_{\ell}^{(1)}=1, a_{r}^{(1)}=3, a_{\ell}^{(2)}=2, a_{r}^{(2)}=0 .
\end{array}
$$

The last two sectors correspond to two degenerate sets of energy eigenvalues.

The total momentum of each state is determined from equation (2.6), and can be expressed in terms of $\left\{I_{j}^{\alpha}\right\}$ using the logarithmic Bethe equations (A.1). Taking the logarithm of equation (2.6) and using the definitions (A.2), the total momentum can be written as

$$
\begin{aligned}
P \equiv \frac{1}{2} \sum_{j=1}^{m_{+}} t_{+}\left(\lambda_{j}^{+}\right)+ & \frac{1}{2} \sum_{j=1}^{m_{-}}\left(t_{-}\left(\lambda_{j}^{-}\right)+2 \pi\right)+\frac{1}{2} \sum_{j=1}^{m_{2 s}} t_{2 s}\left(\lambda_{j}^{2 s}\right) \\
& +\frac{1}{2} \sum_{j=1}^{m_{-2 s}} t_{-2 s}\left(\lambda_{j}^{-2 s}\right)+\frac{1}{2} \sum_{j=1}^{m_{n s}} t_{n s}\left(\lambda_{j}^{n s}\right)(\bmod 2 \pi) .
\end{aligned}
$$

We sum the logarithmic Bethe equations (A.1) over $j$ and $\alpha$. The sum over the functions $\Theta_{\alpha \beta}$ vanishes since they are odd functions. We are left with a sum over the integers:

$$
P \equiv \frac{2 \pi}{M}\left(\frac{1}{2} \sum_{j=1}^{m_{+}} I_{j}^{+}+\frac{1}{2} \sum_{j=1}^{m_{-}}\left(I_{j}^{-}+M\right)+\frac{1}{2} \sum_{j=1}^{m_{2 s}} I_{j}^{2 s}+\frac{1}{2} \sum_{j=1}^{m_{-2 s}} I_{j}^{-2 s}+\sum_{j=1}^{m_{n s}} I_{j}^{n s}\right)(\bmod 2 \pi) .
$$

In order to express the momentum in terms of three independent sets of integers, we note that for the sector $Q=0$, as well as for the sectors corresponding to equations (A.10)-(A.12), where the (half-) integers are chosen from a symmetric interval about zero, the two sets of (half-) integers $\left\{I_{j}^{+}\right\}$and $\left\{-I_{j}^{2 s}\right\}$ fill this interval, and similarly for the sets $\left\{I_{j}^{-}\right\}$and $\left\{-I_{j}^{-2 s}\right\}$. Therefore,

$$
\sum_{j=1}^{m_{ \pm 2 s}} I_{j}^{ \pm 2 s}-\sum_{j=1}^{m_{ \pm}} I_{j}^{ \pm}=0
$$

and the total momentum of a state may be written (using $\left.m_{+} \equiv m_{-}(\bmod 2)\right)$ as

$$
P \equiv \frac{2 \pi}{M}\left(\sum_{j=1}^{m_{+}} \bar{I}_{j}^{+}+\sum_{j=1}^{m_{-2 s}} I_{j}^{-2 s}+\sum_{j=1}^{m_{n s}} I_{j}^{n s}\right)(\bmod 2 \pi)
$$


where $\bar{I}_{j}^{+}=I_{j}^{+}+M / 2$.

However, for the sectors corresponding to equations (A.13)-A.14) there is an additional term involved, since the integer ranges are not symmetric about zero, and there is an offset between the sets $\left\{I_{j}^{ \pm}\right\}$and $\left\{-I_{j}^{ \pm 2 s}\right\}$. In fact, for the sector (A.13) the following relation between the integers holds [21]:

$$
I_{j}^{2 s h}=-I_{j}^{+}+\frac{1}{2}, \quad I_{j}^{-h}=-I_{j}^{-2 s}-\frac{1}{2},
$$

where the superscript $h$ refers to "holes", namely the (half-) integers missing from the set $\left\{I_{j}^{\alpha}\right\}$. The number of $2 s$-holes is $m_{+}$, and the number of '-'-holes is $m_{-2 s}$. The ranges of integers are chosen such that:

$$
\sum_{j=1}^{m_{2 s}} I_{j}^{2 s}+\sum_{j=1}^{m_{+}} I_{j}^{2 s h}=0
$$

that is the $I_{j}^{2 s}$ are chosen from a symmetric range. This is not the case for the $I_{j}^{-}$, which are chosen from the range:

$$
-\frac{1}{2}\left(m_{-}+m_{-2 s}\right) \leq I_{j}^{-} \leq \frac{1}{2}\left(m_{-}+m_{-2 s}-2\right),
$$

so that

$$
\sum_{j=1}^{m_{-}} I_{j}^{-}+\sum_{j=1}^{m_{-2 s}} I_{j}^{-h}=-\frac{1}{2}\left(m_{-}+m_{-2 s}\right) .
$$

Putting equations (A.19)-(A.24) together, we find that for this sector

$$
P \equiv \frac{2 \pi}{M}\left(\sum_{j=1}^{m_{+}} \bar{I}_{j}^{+}+\sum_{j=1}^{m_{-2 s}} I_{j}^{-2 s}+\sum_{j=1}^{m_{n s}} I_{j}^{n s}-\left(\frac{1}{2} m_{n s}+m_{-}+m_{-2 s}\right)\right)(\bmod 2 \pi) .
$$

For the sector corresponding to equation (A.14) we have

$$
I_{j}^{2 s h}=-I_{j}^{+}-\frac{1}{2}, \quad I_{j}^{-h}=-I_{j}^{-2 s}+\frac{1}{2} .
$$

Equation (A.20) still holds, but the range of $I_{j}^{-}$is now such that

$$
\sum_{j=1}^{m_{-}} I_{j}^{-}+\sum_{j=1}^{m_{-2 s}} I_{j}^{-h}=\frac{1}{2}\left(m_{-}+m_{-2 s}\right) .
$$

Therefore the total momentum in this sector is found to be

$$
P \equiv \frac{2 \pi}{M}\left(\sum_{j=1}^{m_{+}} \bar{I}_{j}^{+}+\sum_{j=1}^{m_{-2 s}} I_{j}^{-2 s}+\sum_{j=1}^{m_{n s}} I_{j}^{n s}+\left(\frac{1}{2} m_{n s}+m_{-}+m_{-2 s}\right)\right)(\bmod 2 \pi) .
$$




\section{References}

[1] H.N.V. Temperley and E.H. Lieb, Proc. Roy. Soc. London A322 (1971) 251.

[2] R.J. Baxter, J. Phys. C6 (1973) L445.

[3] R.J. Baxter, Exactly solved models in statistical mechanics (Academic Press, London, 1982).

[4] R. Kedem and B.M. McCoy, J. Stat. Phys. (in press), hep-th/9210129.

[5] G. Albertini, S. Dasmahapatra and B. M. McCoy, Phys. Lett. A170 (1992) 397.

[6] R.J. Baxter and P.A. Pearce, J. Phys. A15 (1982) 897.

[7] V.V. Bazhanov and N.Yu. Reshetikhin, Int. J. Mod. Phys. A4 (1989) 115.

[8] G. Albertini, B.M. McCoy and J.H.H. Perk, Phys. Lett. A 135 (1989) 159, and in Advanced Studies in Pure Mathematics 19 ed. M. Jimbo, T. Miwa and A. Tsuchiya (Kinokuniya-Academic, Tokyo, 1989) p. 1.

[9] V.V. Bazhanov and Yu.G. Stroganov, J. Stat. Phys. 59 (1990) 799.

[10] R.J. Baxter, V.V. Bazhanov and J.H.H. Perk, Int. J. Mod. Phys. B4 (1990) 803.

[11] G. Albertini, J. Phys. A25 (1992) 1799.

[12] P.A. Pearce, Int. J. Mod. Phys. A7, Suppl.1B (1992) 791.

[13] J. Lepowsky and M. Primc, Structure of the standard modules for the affine Lie algebra $A_{1}^{(1)}$, Contemporary Mathematics, Vol. 46 (AMS, Providence, 1985).

[14] A. Rocha-Caridi, in Vertex Operators in Mathematics and Physics, ed. J. Lepowsky, S. Mandelstam and I.M. Singer (Springer, Berlin, 1985) p. 451.

[15] Vl.S. Dotsenko, Nucl. Phys. B235[FS11] (1984) 54.

[16] A.B. Zamolodchikov and V.A. Fateev, Sov. Phys. JETP 62 (1985) 215.

[17] J.L. Cardy, Nucl. Phys. B275[FS17] (1986) 200.

[18] R. Kedem, T.R. Klassen, B.M. McCoy, and E. Melzer, Phys. Lett. B (in press), hepth/9301046.

[19] R. Kedem, J. Stat. Phys. (in press), hep-th/9210146.

[20] D. Gepner and Z. Qiu, Nucl. Phys. B285 [FS19] (1987) 423.

[21] G. Albertini, S. Dasmahapatra and B.M. McCoy, Int. J. Mod. Phys. A7, Suppl. 1A (1992) 1.

[22] A. Klümper and P.A. Pearce, J. Stat. Phys. 64 (1991) 13; Physica A 183 (1992) 304.

[23] R.P. Stanley, Ordered structures and partitions, Mem. Amer. Math. Soc. 119 (1972).

[24] G.E. Andrews, The Theory of Partitions (Addison-Wesley, London, 1976).

[25] V.G. Kac and D.H. Peterson, Adv. in Math. 53 (1984) 125. 
[26] M. Jimbo and T. Miwa, Adv. Stud. in Pure Math. 4 (1984) 97.

[27] P. Goddard, A. Kent and D. Olive, Commun. Math. Phys. 103 (1986) 105.

[28] B.L. Feigin and D.B. Fuchs, Funct. Anal. Appl. 17 (1983) 241.

[29] G. Felder, Nucl. Phys. B317 (1989) 215.

[30] A.A. Belavin, A.M. Polyakov and A.B. Zamolodchikov, J. Stat. Phys. 34 (1984) 763; Nucl. Phys. B241 (1984) 337.

[31] V.A. Fateev and A.B. Zamolodchikov, Nucl. Phys. B280[FS18] (1987) 644.

[32] D. Altschuler, M. Bauer and H. Saleur, J. Phys. A23 (1990) L789.

[33] J. Distler and Z. Qiu, Nucl. Phys. B336 (1990) 533.

[34] R. Kedem, T.R. Klassen, B.M. McCoy and E. Melzer, Phys. Lett. B (in press), hepth/9211102.

[35] A.B. Zamolodchikov, in Advanced Studies in Pure Mathematics 19 ed. M. Jimbo, T. Miwa and A. Tsuchiya (Kinokuniya-Academic, Tokyo, 1989) p. 641.

[36] A.B. Zamolodchikov, Int. J. Mod. Phys. A3 (1988) 743.

[37] R. Köberle and J.A. Swieca, Phys. Lett. B86 (1979) 209.

[38] R.J. Baxter, J. Phys. A13 (1980) L61; J. Stat. Phys. 26 (1981) 427.

[39] Kashiwara and Miwa, Nucl. Phys. B275 (1986) 121.

[40] V. Pasquier, J. Phys. A20 (1987) L217 and L221.

[41] G.E. Andrews, R.J. Baxter and P.J. Forrester, J. Stat. Phys. 35 (1984) 193.

[42] P. Fendley and P. Ginsparg, Nucl. Phys. B324 (1989) 549.

[43] A.B. Zamolodchikov, Sov. J. Nucl. Phys. 46 (1987) 1090.

[44] A. Ludwig and J.L. Cardy, Nucl. Phys. B285 [FS19] (1987) 687.

[45] A.B. Zamolodchikov, Landau Institute preprint (1989).

[46] D. Bernard and A. LeClair, Nucl. Phys. B340 (1990) 721.

[47] N.Yu. Reshetikhin and F.A. Smirnov, Commun. Math. Phys. 131 (1990) 157.

[48] Al.B. Zamolodchikov, Nucl. Phys. B358 (1991) 497 and 524.

[49] V.A. Fateev and Al.B. Zamolodchikov, Phys. Lett. B271 (1991) 91.

[50] T.R. Klassen and E. Melzer, Nucl. Phys. B370 (1992) 511.

[51] F. Ravanini, Phys. Lett. B274 (1992) 345.

[52] T.R. Klassen and E. Melzer, Nucl. Phys. B (in press), hep-th/9110047.

[53] V.V. Bazhanov and N.Yu. Reshetikhin, Progr. Theor. Phys. Suppl. 102 (1990) 301.

[54] J.L. Cardy, in Fields, strings, and critical phenomena, Les Houches 1988, ed. E. Brézin and J. Zinn-Justin (North-Holland, Amsterdam, 1989). 
Table 2: The first terms of the partition function in the sector $Q=0$ and $C=1$, where $m_{-}$is even, and $m_{+}=2 m_{n s}+3 m_{-}+4 m_{-2 s}$. The momentum ranges are given in equations (3.2) -(3.4). The sum of the momenta in the square brackets gives the total momentum, and thus the power of $q$, listed on the left. $N$ is the number of states with given $m_{\alpha}$ and fixed total momentum, whose overall number is listed on the right. These are the coefficients of $q^{n}$ in the power expansion of $\widehat{\chi}_{0}$.

\begin{tabular}{|c|c|c|c|c|c|c|c|c|c|}
\hline Order & $m_{+}$ & $m_{-2 s}$ & $m_{-}$ & $m_{n s}$ & $P_{\min }^{+,-2 s}$ & $P_{\min }^{n s}$ & {$\left[P^{n s} ; P^{-2 s} ; P^{+}\right]\left(\right.$Units of $\left.\frac{\pi}{M}\right)$} & $N$ & Tot \\
\hline 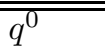 & $\overline{0}$ & 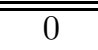 & 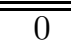 & 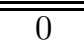 & 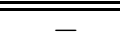 & 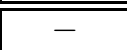 & {$[-[-;-;-]$} & 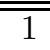 & 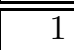 \\
\hline$q^{2}$ & 2 & 0 & $\overline{0}$ & 1 & $\pi / M$ & 0 & {$[0 ;-; 1,3]$} & 1 & 1 \\
\hline$q^{3}$ & 2 & 0 & 0 & 1 & $\pi / M$ & 0 & {$[0 ;-; 1,5]$} & 1 & 1 \\
\hline$q^{4}$ & 2 & 0 & 0 & 1 & $\pi / M$ & 0 & {$[0 ;-; 1,7],[0 ;-; 3,5]$} & 2 & 2 \\
\hline$q^{5}$ & 2 & 0 & 0 & 1 & $\pi / M$ & 0 & {$[0 ;-; 1,9],[0 ;-; 3,7]$} & 2 & 2 \\
\hline \multirow[t]{2}{*}{$q^{6}$} & 2 & $\overline{0}$ & $\overline{0}$ & 1 & $\pi / M$ & 0 & {$[0 ;-; 1,11],[0 ;-; 3,9],[0 ;-; 5,7]$} & 3 & \\
\hline & 4 & 1 & 0 & 0 & 0 & - & {$[-; 0 ; 0,2,4,6]$} & 1 & 4 \\
\hline \multirow[t]{2}{*}{$q^{7}$} & 2 & $\overline{0}$ & $\overline{0}$ & 1 & $\pi / M$ & 0 & {$[0 ;-; 1,13],[0 ;-; 3,11],[0 ;-; 5,9]$} & 3 & \\
\hline & 4 & 1 & 0 & 0 & 0 & - & {$[-; 0 ; 0,2,4,8]$} & 1 & 4 \\
\hline \multirow[t]{4}{*}{$q^{8}$} & 2 & 0 & 0 & 1 & $\pi / M$ & 0 & {$[0 ;-; 1,15],[0 ;-; 3,13]$} & & \\
\hline & & & & & & & {$[0 ;-; 5,11],[0 ;-; 7,9]$} & 4 & \\
\hline & 4 & 1 & 0 & 0 & 0 & - & {$[-; 0 ; 0,2,4,10],[-; 0 ; 0,2,6,8]$} & 2 & \\
\hline & 4 & 0 & 0 & 2 & $\pi / M$ & $-\pi / M$ & {$[-1,1 ;-; 1,3,5,7]$} & 1 & 7 \\
\hline \multirow[t]{4}{*}{$q^{9}$} & 2 & 0 & 0 & 1 & $\pi / M$ & 0 & {$[0 ;-; 1,17],[0 ;-; 3,15]$} & & \\
\hline & & & & & & & {$[0 ;-; 5,13],[0 ;-; 7,11]$} & 4 & \\
\hline & 4 & 1 & 0 & 0 & 0 & - & $\begin{array}{l}{[-; 0 ; 0,2,4,12],[-; 0 ; 0,2,6,10]} \\
{[-; 0 ; 0,4,6,8]}\end{array}$ & 3 & \\
\hline & 4 & 0 & 0 & 2 & $\pi / M$ & $-\pi / M$ & {$[-1,1 ;-; 1,3,5,9]$} & 1 & 8 \\
\hline \multirow[t]{5}{*}{$q^{10}$} & 2 & 0 & 0 & 1 & $\pi / M$ & 0 & {$[0 ;-; 1,19],[0 ;-; 3,17]$} & & \\
\hline & & & & & & & {$[0 ;-; 5,15],[0 ;-; 7,13],[0 ;-;$} & 5 & \\
\hline & 4 & 1 & 0 & 0 & 0 & - & $\begin{array}{l}{[-; 0 ; 0,2,4,14],[-; 0 ; 0,2,6,12]} \\
{[-: 0 ; 0.4 .6 .10] .[-; 0: 0.28 .10]}\end{array}$ & & \\
\hline & & & & & & & {$[-; 0 ; 2,4,6,8]$} & 5 & \\
\hline & 4 & 0 & 0 & 2 & $\pi / M$ & $-\pi / M$ & {$[-1,1 ;-; 1,3,5,11],[-1,1 ;-; 1,3,7,9]$} & 2 & 12 \\
\hline \multirow[t]{6}{*}{$q^{11}$} & 2 & 0 & 0 & 1 & $\pi / M$ & 0 & {$[0 ;-; 1,21],[0 ;-; 3,19]$} & & \\
\hline & & & & & & & {$[0 ;-; 5,17],[0 ;-; 7,15],[0 ;-; 9,13]$} & 5 & \\
\hline & 4 & 1 & 0 & 0 & 0 & - & {$[-; 0 ; 0,2,4,16],[-; 0 ; 0,2,6,14]$} & & \\
\hline & & & & & & & {$[-; 0 ; 0,2,8,12],[-; 0 ; 0,4,6,12]$} & & \\
\hline & & & & & & & {$[-; 0 ; 0,4,8,10],[-; 0 ; 2,4,6,10]$} & 6 & \\
\hline & 4 & 0 & 0 & 2 & $\pi / M$ & $-\pi / M$ & $\begin{array}{l}{[-1,1 ;-; 1,3,5,13],[-1,1 ;-; 1,3,7,11]} \\
{[-1,1 ;-; 1,5,7,9]}\end{array}$ & 3 & 14 \\
\hline \multirow[t]{8}{*}{$q^{12}$} & 2 & 0 & 0 & 1 & $\pi / M$ & 0 & {$[0 ;-; 1,23],[0 ;-; 3,21],[0 ;-; 5,19]$} & 6 & \\
\hline & 4 & 1 & 0 & 0 & 0 & - & {$\left[\begin{array}{l}0 ;-; \\
{[-; 0 ; 0,2,4,18],[-; 0 ; 0,2,6,16]}\end{array}\right.$} & & \\
\hline & & & & & & & {$[-; 0 ; 0,2,8,14],[-; 0 ; 0,2,10,12]$} & & \\
\hline & & & & & & & {$[-; 0 ; 0,4,6,14],[-; 0 ; 2,4,6,12]$} & & \\
\hline & & & & & & & $\begin{array}{l}{[-; 0 ; 0,4,8,12],[-; 0 ; 2,4,8,10]} \\
{[-; 0 ; 0,6,8,10]}\end{array}$ & 9 & \\
\hline & 4 & 0 & 0 & 2 & $\pi / M$ & $-\pi / M$ & {$[-1,1 ;-; 1,3,5,15],[-1,1 ;-; 1,3,7,13]$} & & \\
\hline & & & & & & & $\begin{array}{l}{[-1,1 ;-; 1,5,7,11],[-1,1 ;-; 1,3,9,11]} \\
{[-1,1 ;-; 3,5,7,9]}\end{array}$ & 5 & \\
\hline & 6 & 0 & 2 & 0 & $-\pi / M$ & - & {$[-;-;-1,1,3,5,7,9]$} & 1 & 21 \\
\hline
\end{tabular}


Table 3: The first few terms in the partition function in the sector $Q=0$ and $C=-1$, corresponding to $m_{-}$odd, and $m_{+}=2 m_{n s}+3 m_{-}+4 m_{-2 s}$. The momentum ranges are the same as in table 2. The total number of states on the right corresponds to the first few terms in the expansion of $q^{3} \widehat{\chi}_{3}$.

\begin{tabular}{|c|c|c|c|c|c|c|c|c|c|}
\hline Order & $m_{+}$ & $m_{-2 s}$ & $m_{-}$ & $m_{n s}$ & $P_{\min }^{+,-2 s}$ & $P_{\min }^{n s}$ & {$\left[P^{n s} ; P^{-2 s} ; P^{+}\right]\left(\right.$Units of $\left.\frac{\pi}{M}\right)$} & $N$ & Tot \\
\hline$\overline{q^{3}}$ & 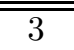 & $\overline{0}$ & $\overline{1}$ & $\overline{\overline{0}}$ & 0 & 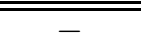 & {$[-;-; 0+2+4]$} & 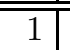 & $\overline{1}$ \\
\hline$q^{4}$ & 3 & 0 & 1 & 0 & 0 & - & {$[-;-; 0+2+6]$} & 1 & 1 \\
\hline$q^{5}$ & 3 & $\overline{0}$ & 1 & $\overline{0}$ & 0 & - & {$[-;-; 0+2+8],[-;-; 0+4+6]$} & 2 & 2 \\
\hline$q^{6}$ & 3 & 0 & 1 & 0 & 0 & - & $\begin{array}{l}{[-;-; 0+2+10],[-;-; 0+4+8]} \\
{[-;-; 2+4+6]}\end{array}$ & 3 & 3 \\
\hline$q^{7}$ & 3 & 0 & 1 & 0 & 0 & $\overline{-}$ & $\begin{array}{l}{[-;-; 0+2+12],[-;-; 0+4+10]} \\
{[-;-; 0+6+8],[-;-; 2+4+8]}\end{array}$ & 4 & 4 \\
\hline$q^{8}$ & 3 & 0 & 1 & 0 & 0 & - & $\begin{array}{l}{[-;-; 0+2+14],[-;-; 0+4+12]} \\
{[-;-; 0+6+10],[-;-; 2+4+10]} \\
{[-;-; 2+6+8]}\end{array}$ & 5 & 5 \\
\hline$q^{9}$ & 3 & 0 & 1 & 0 & 0 & $\begin{array}{c}- \\
-2 \pi / M\end{array}$ & $\begin{array}{l}{[-;-; 0+2+16],[-;-; 0+4+14]} \\
{[-;-; 2+4+12],[-;-; 0+6+12]} \\
{[-;-; 2+6+10],[-;-; 4+6+8]} \\
{[-;-; 0+8+10]} \\
{[-2 ;-; 0+2+4+6+8]}\end{array}$ & $\begin{array}{l}7 \\
1\end{array}$ & 8 \\
\hline$q^{10}$ & 3 & 0 & 1 & 0 & 0 & $\begin{array}{c}- \\
-2 \pi / M\end{array}$ & $\begin{array}{l}{[-;-; 0+2+18],[-;-; 0+4+16]} \\
{[-;-; 2+4+14],[-;-; 0+6+14]} \\
{[-;-; 2+6+12],[-;-; 4+6+10]} \\
{[-;-; 0+8+12],[-;-; 2+8+10]} \\
{[-2 ;-; 0+2+4+6+10]} \\
{[0 ;-; 0+2+4+6+8]}\end{array}$ & $\begin{array}{l}8 \\
2\end{array}$ & 10 \\
\hline$q^{11}$ & 3 & 0 & 1 & 0 & 0 & $-2 \pi / M$ & $\begin{array}{l}{[-;-; 0+2+20],[-;-; 0+4+18]} \\
{[-;-; 2+4+16],[-;-; 0+6+16]} \\
{[-;-; 2+6+14],[-;-; 4+6+12]} \\
{[-;-; 0+8+14],[-;-; 2+8+12]} \\
{[-;-; 4+8+10],[-;-; 0+10+12]} \\
{[-2 ;-; 0+2+4+6+12]} \\
{[-2 ;-; 0+2+4+8+10]} \\
{[0 ;-; 0+2+4+6+10]} \\
{[2 ;-; 0+2+4+6+8]}\end{array}$ & 10 & 14 \\
\hline$q^{12}$ & 3 & 0 & 1 & 0 & 0 & $-2 \pi / M$ & $\begin{array}{l}{[-;-; 0+2+22],[-;-; 0+4+20]} \\
{[-;-; 2+4+18],[-;-; 0+6+18]} \\
{[-;-; 2+6+16],[-;-; 4+6+14]} \\
{[-;-; 0+8+16],[-;-; 2+8+14]} \\
{[-;-; 4+8+12],[-;-; 0+10+14]} \\
{[-;-; 2+10+12],[-;-; 6+8+10]} \\
{[-2 ;-; 0+2+4+6+14]} \\
{[-2 ;-; 0+2+4+8+12]} \\
{[-2 ;-; 0+2+6+8+10]} \\
{[0 ;-; 0+2+4+6+12]} \\
{[0 ;-; 0+2+4+8+10]} \\
{[2 ;-; 0+2+4+6+10]}\end{array}$ & 12 & 18 \\
\hline
\end{tabular}


Table 4: The first few terms for the sector of the partition function corresponding to $Q=0$ and $C=1$, where one of the ' + '-excitations is a left-mover, and the rest are right-movers. This corresponds to $m_{-}$even and $m_{+}=2 m_{n s}+3 m_{-}+4 m_{-2 s}-1$. The momentum ranges are the same as in table 2, and there is an additional term ("shift") in the momentum of $\frac{\pi}{M}\left(m_{-}+m_{-2 s}-1\right)$ which is the momentum of the left-mover. The coefficients on the right correspond to the expansion of $\hat{\chi}_{2 / 5}$.

\begin{tabular}{|c|c|c|c|c|c|c|c|c|c|c|}
\hline Order & $m_{+}$ & $m_{-2 s}$ & $m_{-}$ & $m_{n s}$ & $P_{\min }^{+,-2 s}$ & $P_{\min }^{n s}$ & {$\left[P^{n s} ; P^{-2 s} ; P^{+}\right]\left(\right.$Units of $\left.\frac{\pi}{M}\right)$} & Shift & $N$ & Tot \\
\hline$\overline{q^{0}}$ & $\overline{1} 1$ & ("0 & "0 & $\overline{1} 1$ & 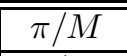 & "0 & 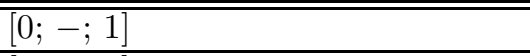 & $-\pi / M$ & 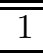 & $\overline{1} 1$ \\
\hline$q^{1}$ & 1 & 0 & 0 & 1 & $\pi / M$ & 0 & {$[0 ;-; 3]$} & $-\pi / M$ & 1 & 1 \\
\hline$q^{2}$ & 1 & 0 & 0 & 1 & $\pi / M$ & 0 & {$[0 ;-; 5]$} & $-\pi / M$ & 1 & 1 \\
\hline \multirow[t]{2}{*}{$q^{3}$} & 1 & 0 & 0 & 1 & $\pi / M$ & 0 & {$[0 ;-; 7]$} & $-\pi / M$ & 1 & \\
\hline & 3 & 1 & 0 & 0 & 0 & - & {$[-; 0 ; 0,2,4]$} & 0 & 1 & 2 \\
\hline \multirow[t]{3}{*}{$q^{4}$} & 1 & 0 & 0 & 1 & $\pi / M$ & 0 & {$[0 ;-; 9]$} & $-\pi / M$ & 1 & \\
\hline & 3 & 1 & 0 & 0 & 0 & - & {$[-; 0 ; 0,2,6]$} & 0 & 1 & \\
\hline & 3 & 0 & 0 & 2 & $\pi / M$ & $-\pi / M$ & {$[-1,1 ;-; 1,3,5]$} & $-\pi / M$ & 1 & 3 \\
\hline \multirow[t]{3}{*}{$\overline{q^{5}}$} & 1 & 0 & 0 & 1 & $\pi / M$ & 0 & {$[0 ;-; 11]$} & $-\pi / M$ & 1 & \\
\hline & 3 & 1 & 0 & 0 & 0 & - & {$[-; 0 ; 0,2,8],[-; 0 ; 0,4,6]$} & 0 & 2 & \\
\hline & 3 & 0 & 0 & 2 & $\pi / M$ & $-\pi / M$ & {$[-1,1 ;-; 1,3,7]$} & $-\pi / M$ & 1 & 4 \\
\hline \multirow[t]{4}{*}{$q^{6}$} & 1 & 0 & 0 & 1 & $\pi / M$ & 0 & {$[0 ;-; 13]$} & $-\pi / M$ & 1 & \\
\hline & 3 & 1 & 0 & 0 & 0 & - & {$[-; 0 ; 0,2,10],[-; 0 ; 0,4,8]$} & & & \\
\hline & & & & & & & {$[-; 0 ; 2,4,6]$} & 0 & 3 & \\
\hline & 3 & 0 & 0 & 2 & $\pi / M$ & $-\pi / M$ & {$[-1,1 ;-; 1,3,9],[-1,1 ;-; 1,5,7]$} & $-\pi / M$ & 2 & 6 \\
\hline \multirow[t]{5}{*}{$q^{7}$} & 1 & 0 & 0 & 1 & $\pi / M$ & 0 & {$[0 ;-; 15]$} & $-\pi / M$ & 1 & \\
\hline & 3 & 1 & 0 & 0 & 0 & - & {$[-; 0 ; 0,2,12],[-; 0 ; 0,4,10]$} & & & \\
\hline & & & & & & & {$[-; 0 ; 2,4,8],[-; 0 ; 0,6,8]$} & 0 & 4 & \\
\hline & 3 & 0 & 0 & 2 & $\pi / M$ & $-\pi / M$ & {$[-1,1 ;-; 1,3,11],[-1,1 ;-; 1,5,9]$} & & & \\
\hline & & & & & & & {$[-1,1 ;-; 3,5,7]$} & $-\pi / M$ & 3 & 8 \\
\hline \multirow[t]{7}{*}{$q^{8}$} & 1 & 0 & 0 & 1 & $\pi / M$ & 0 & {$[0 ;-; 17]$} & $-\pi / M$ & 1 & \\
\hline & 3 & 1 & 0 & 0 & 0 & - & {$[-; 0 ; 0,2,14],[-; 0 ; 0,4,12]$} & & & \\
\hline & & & & & & & {$[-; 0 ; 2,4,10],[-; 0 ; 0,6,10]$} & & & \\
\hline & & & & & & & {$[-; 0 ; 2,6,8]$} & 0 & 5 & \\
\hline & 3 & 0 & 0 & 2 & $\pi / M$ & $-\pi / M$ & {$[-1,1 ;-; 1,3,13],[-1,1 ;-; 1,5,11]$} & & & \\
\hline & & & & & & & {$[-1,1 ;-; 3,5,9],[-1,1 ;-; 1,7,9]$} & $-\pi / M$ & 4 & \\
\hline & 5 & 0 & 2 & 0 & $-\pi / M$ & - & {$[-;-;-1,1,3,5,7]$} & $\pi / M$ & 1 & 11 \\
\hline \multirow[t]{8}{*}{$q^{9}$} & 1 & 0 & 0 & 1 & $\pi / M$ & 0 & {$[0 ;-; 19]$} & $-\pi / M$ & 1 & \\
\hline & 3 & 1 & 0 & 0 & 0 & - & {$[-; 0 ; 0,2,16],[-; 0 ; 0,4,14]$} & & & \\
\hline & & & & & & & {$[-; 0 ; 2,4,12],[-; 0 ; 0,6,12]$} & & & \\
\hline & & & & & & & $\begin{array}{l}{[-; 0 ; 2,6,10],[-; 0 ; 4,6,8]} \\
{[-; 0 ; 0,8,10],}\end{array}$ & 0 & 7 & \\
\hline & 3 & 0 & 0 & 2 & $\pi / M$ & $-\pi / M$ & $\begin{array}{l}{[-1,1 ;-; 1,3,15],[-1,1 ;-; 1,5,13]} \\
{[-1,1 ;-; 3,5,11],[-1,1 ;-; 1,7,11]}\end{array}$ & & & \\
\hline & & & & & & & {$[-1,1 ;-; 3,7,9]$} & $-\pi / M$ & 5 & \\
\hline & 5 & 0 & 2 & 0 & $-\pi / M$ & - & {$[-;-;-1,1,3,5,9]$} & $\pi / M$ & 1 & \\
\hline & 5 & 1 & 0 & 1 & 0 & $-2 \pi / M$ & {$[-2 ; 0 ; 0,2,4,6,8]$} & 0 & 1 & 15 \\
\hline
\end{tabular}


Table 5: The first few terms in the partition function in the sector $Q=0, C=-1$ where one '+'-excitation is left-moving and all the rest are right-movers. The shift and $m_{+}$are as in table 4 , and the momentum ranges are as in table 2. The coefficients on the right correspond to the expansion of $q \widehat{\chi}_{7 / 5}$.

\begin{tabular}{|c|c|c|c|c|c|c|c|c|c|c|}
\hline Order & $m_{+}$ & $m_{-2 s}$ & $m_{-}$ & $m_{n s}$ & $P_{\min }^{+,-2 s}$ & $P_{\min }^{n s}$ & {$\left[P^{n s} ; P^{-2 s} ; P^{+}\right]\left(\right.$Units of $\left.\frac{\pi}{M}\right)$} & Shift & $N$ & Tot \\
\hline$q^{1}$ & $\overline{2}$ & $\overline{0}$ & $\overline{1}$ & $\overline{0}$ & $\overline{0}$ & - & $\overline{[-;-; 0,2]}$ & 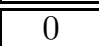 & 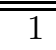 & 1 \\
\hline$q^{2}$ & 2 & 0 & 1 & 0 & 0 & - & {$[-;-; 0,4]$} & 0 & 1 & 1 \\
\hline$q^{3}$ & 2 & 0 & 1 & 0 & 0 & - & {$[-;-; 0,6],[-;-; 2,4]$} & 0 & 2 & 2 \\
\hline$q^{4}$ & 2 & 0 & 1 & 0 & 0 & - & {$[-;-; 0,8],[-;-; 2,6]$} & 0 & 2 & 2 \\
\hline \multirow[t]{2}{*}{$\overline{q^{5}}$} & 2 & 0 & 1 & 0 & 0 & - & {$[-;-; 0,10],[-;-; 2,8],[-;-; 4,6]$} & 0 & 3 & \\
\hline & 4 & 0 & 1 & 1 & 0 & $-2 \pi / M$ & {$[-2 ;-; 0,2,4,6]$} & 0 & 1 & 4 \\
\hline \multirow[t]{2}{*}{$q^{6}$} & 2 & 0 & 1 & 0 & 0 & - & {$[-;-; 0,12],[-;-; 2,10],[-;-; 4,8]$} & 0 & 3 & \\
\hline & 4 & 0 & 1 & 1 & 0 & $-2 \pi / M$ & {$[-2 ;-; 0,2,4,8],[0 ;-; 0,2,4,6]$} & 0 & 2 & 5 \\
\hline \multirow[t]{3}{*}{$q^{7}$} & 2 & 0 & 1 & 0 & 0 & - & {$[-;-; 0,14],[-;-; 2,12],[-;-; 4,10]$} & & & \\
\hline & & & & & & & {$[-;-; 6,8]$} & 0 & 4 & \\
\hline & 4 & 0 & 1 & 1 & 0 & $-2 \pi / M$ & $\begin{array}{l}{[-2 ;-; 0,2,4,10],[-2 ;-; 0,2,6,8]} \\
{[0 ;-; 0,2,4,8],[2 ;-; 0,2,4,6]}\end{array}$ & 0 & 4 & 8 \\
\hline \multirow[t]{4}{*}{$q^{8}$} & 2 & 0 & 1 & 0 & 0 & - & {$[-;-; 0,16],[-;-; 2,14],[-;-; 4,12]$} & & & \\
\hline & 4 & & & 1 & 0 & $-2 \pi / M$ & {$[-;-; 6,10]\left[\begin{array}{l}{[-2.02412][-2 .-.02610]} \\
{[-2}\end{array}\right.$} & 0 & 4 & \\
\hline & & & & & & $2 \pi / \Delta \Delta$ & {$[-2 ;-; 0,4,6,8],[0 ;-; 0,2,4,10]$} & & & \\
\hline & & & & & & & {$[0 ;-; 0,2,6,8],[2 ;-; 0,2,4,8]$} & 0 & 6 & 10 \\
\hline \multirow[t]{5}{*}{$q^{9}$} & 2 & 0 & 1 & 0 & 0 & - & {$[-;-; 0,18],[-;-; 2,16],[-;-; 4,14]$} & & & \\
\hline & 4 & 0 & 1 & 1 & 0 & $-2 \pi / M$ & $\begin{array}{l}{[-;-; 0,12],[-;-; 8,10]} \\
{[-2 ;-; 0,2,4,14],[-2 ;-; 0,2,6,12]}\end{array}$ & 0 & 5 & \\
\hline & & & & & & & {$[-2 ;-; 0,4,6,10],[-2 ;-; 2,4,6,8]$} & & & \\
\hline & & & & & & & $\begin{array}{l}{[-2 ;-; 0,2,8,10],[0 ;-; 0,2,4,12]} \\
{[0 ;-; 0,2,6,10],[0 ;-; 0,4,6,8]}\end{array}$ & & & \\
\hline & & & & & & & {$[2 ;-; 0,2,4,10],[2 ;-; 0,2,6,8]$} & 0 & 10 & 15 \\
\hline \multirow[t]{7}{*}{$q^{10}$} & 2 & 0 & 1 & 0 & 0 & - & {$[-;-; 0,20],[-;-; 2,18],[-;-; 4,16]$} & & & \\
\hline & & & & 1 & 0 & $-2 \pi / M$ & 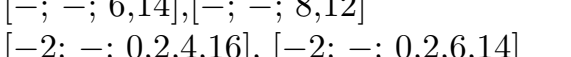 & 0 & 5 & \\
\hline & 4 & & & & & & {$[-2 ;-; 0,4,6,12],[-2 ;-; 2,4,6,10]$} & & & \\
\hline & & & & & & & {$[-2 ;-; 0,2,8,12],[-2 ;-; 0,4,8,10]$} & & & \\
\hline & & & & & & & {$[0 ;-; 0,2,4,14],[0 ;-; 0,2,6,12]$} & & & \\
\hline & & & & & & & {$[0 ;-; 0,4,6,10],[0 ;-; 2,4,6,8]$} & & & \\
\hline & & & & & & & $\begin{array}{l}{[0 ;-; 0,2,8,10],[2 ;-; 0,2,4,12]} \\
{[2 ;-; 0,2,6,10],[2 ;-; 0,4,6,8]}\end{array}$ & 0 & 14 & 19 \\
\hline
\end{tabular}


Table 6: The first terms in the partition function for the sector $Q=1$ and $m_{-}-m_{++}=1$, where $m_{+}=2 m_{n s}+3 m_{-}+4 m_{-2 s}-2$, and the momentum ranges are: $-\frac{\pi}{M}\left(m_{-2 s}+m_{-}-3\right) \leq$ $P_{j}^{+}<\infty,-\frac{\pi}{M}\left(m_{-2 s}+m_{-}-3\right) \leq P_{j}^{-2 s} \leq \frac{\pi}{M}\left(m_{-2 s}+m_{-}-3\right),-\frac{\pi}{M}\left(m_{n s}+2 m_{-2 s}+2 m_{-}-3\right) \leq$ $P_{j}^{n s} \leq \frac{\pi}{M}\left(m_{n s}+2 m_{-2 s}+2 m_{-}-3\right)$.

\begin{tabular}{|c|c|c|c|c|c|c|c|c|c|}
\hline Order & $m_{+}$ & $m_{-2 s}$ & $m_{-}$ & $m_{n s}$ & $P_{\min }^{+,-2 s}$ & $P_{\min }^{n s}$ & {$\left[P^{n s} ; P^{-2 s} ; P^{+}\right]\left(\right.$Units of $\left.\frac{\pi}{M}\right)$} & $N$ & Tot \\
\hline 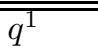 & 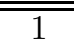 & $\overline{0}$ & 1 & 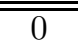 & $2 \pi / M$ & 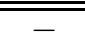 & $\overline{[-;-; 2]}$ & $\bar{~} 1$ & $\overline{1}$ \\
\hline$q^{2}$ & 1 & 0 & 1 & 0 & $2 \pi / M$ & - & {$[-;-; 4]$} & 1 & 1 \\
\hline$q^{3}$ & 1 & 0 & 1 & 0 & $2 \pi / M$ & $\overline{-}$ & {$[-;-; 6]$} & 1 & 1 \\
\hline$q^{4}$ & 1 & 0 & 1 & 0 & $2 \pi / M$ & - & {$[-;-; 8]$} & 1 & 1 \\
\hline$q^{5}$ & 1 & 0 & 1 & 0 & $2 \pi / M$ & - & {$[-;-; 10]$} & 1 & 1 \\
\hline \multirow[t]{2}{*}{$q^{6}$} & 1 & 0 & 1 & 0 & $2 \pi / M$ & - & {$[-;-; 12]$} & 1 & \\
\hline & 3 & 0 & 1 & 1 & $2 \pi / M$ & 0 & {$[0 ;-; 2,4,6]$} & 1 & 2 \\
\hline \multirow[t]{2}{*}{$q^{7}$} & 1 & 0 & 1 & 0 & $2 \pi / M$ & - & {$[-;-; 14]$} & 1 & \\
\hline & 3 & 0 & 1 & 1 & $2 \pi / M$ & 0 & {$[0 ;-; 2,4,8]$} & 1 & 2 \\
\hline \multirow[t]{3}{*}{$q^{8}$} & 1 & 0 & 1 & 0 & $2 \pi / M$ & - & {$[-;-; 16]$} & 1 & \\
\hline & 3 & 0 & 1 & 1 & $2 \pi / M$ & 0 & {$[0 ;-; 2,4,10],[0 ;-; 2,6,8]$} & 2 & \\
\hline & 4 & 0 & 2 & 0 & $\pi / M$ & - & {$[-;-; 1,3,5,7]$} & 1 & 4 \\
\hline \multirow[t]{3}{*}{$q^{9}$} & 1 & $\overline{0}$ & 1 & $\overline{0}$ & $2 \pi / M$ & - & {$[-;-; 18]$} & 1 & \\
\hline & 3 & 0 & 1 & 1 & $2 \pi / M$ & 0 & {$[0 ;-; 2,4,12],[0 ;-; 2,6,10],[0 ;-; 4,6,8]$} & 3 & \\
\hline & 4 & 0 & 2 & 0 & $\pi / M$ & - & {$[-;-; 1,3,5,9]$} & 1 & 5 \\
\hline \multirow[t]{4}{*}{$q^{10}$} & 1 & 0 & 1 & $\overline{0}$ & $2 \pi / M$ & - & {$[-;-; 20]$} & 1 & \\
\hline & 3 & 0 & 1 & 1 & $2 \pi / M$ & 0 & {$[0 ;-; 2,4,14],[0 ;-; 2,6,12],[0 ;-; 4,6,10]$} & & \\
\hline & & & & & & & {$[0 ;-; 2,8,10]$} & 4 & \\
\hline & 4 & 0 & 2 & 0 & $\pi / M$ & - & {$[-;-; 1,3,5,11],[-;-; 1,3,7,9]$} & 2 & 7 \\
\hline \multirow[t]{4}{*}{$q^{11}$} & 1 & 0 & 1 & 0 & $2 \pi / M$ & - & {$[-;-; 22]$} & 1 & \\
\hline & 3 & 0 & 1 & 1 & $2 \pi / M$ & 0 & {$[0 ;-; 2,4,16],[0 ;-; 2,6,14],[0 ;-; 4,6,12]$} & & \\
\hline & & 0 & & & $\pi / M$ & & $\begin{array}{l}{[0 ;-; 2,8,12],[0 ;-; 4,8,10]} \\
[-;-; 13.5,13] .-:-: 13,7,11]\end{array}$ & 5 & \\
\hline & 4 & 0 & 2 & 0 & & - & $\begin{array}{l}{[-;-; 1,0,0,10],[-;-; 1,0, \imath, 11]} \\
{[-;-; 1,5,7,9]}\end{array}$ & 3 & 9 \\
\hline \multirow[t]{5}{*}{$q^{12}$} & 1 & 0 & 1 & 0 & $2 \pi / M$ & - & {$[-;-; 24]$} & 1 & \\
\hline & 3 & 0 & 1 & 1 & $2 \pi / M$ & 0 & {$[0 ;-; 2,4,18],[0 ;-; 2,6,16],[0 ;-; 4,6,14]$} & & \\
\hline & & & & & & & $\begin{array}{l}{[0 ;-; 2,8,14],[0 ;-; 4,8,12],[0 ;-; 2,10,12]} \\
{[0 ;-; 6.8,10]}\end{array}$ & 7 & \\
\hline & 4 & 0 & 2 & 0 & $\pi / M$ & - & {$[-;-; 1,3,5,15],[-;-; 1,3,7,13]$} & & \\
\hline & & & & & & & {$[-;-; 3,5,7,9]$} & 5 & 13 \\
\hline
\end{tabular}


Table 7: The first terms in the partition function for the sector $Q=1$ and $m_{-}-m_{++}=-1$, where $m_{+}=2 m_{n s}+3 m_{-}+4 m_{-2 s}+2$, and the momentum ranges are: $-\frac{\pi}{M}\left(m_{-2 s}+m_{-}-1\right) \leq$ $P_{j}^{+}<\infty,-\frac{\pi}{M}\left(m_{-2 s}+m_{-}-1\right) \leq P_{j}^{-2 s} \leq \frac{\pi}{M}\left(m_{-2 s}+m_{-}-1\right),-\frac{\pi}{M}\left(m_{n s}+2 m_{-2 s}+2 m_{-}-1\right) \leq$ $P_{j}^{n s} \leq \frac{\pi}{M}\left(m_{n s}+2 m_{-2 s}+2 m_{-}-1\right)$.

\begin{tabular}{|c|c|c|c|c|c|c|c|c|c|}
\hline Order & $m_{+}$ & $m_{-2 s}$ & $m_{-}$ & $m_{n s}$ & $P_{\text {min }}^{+,-2 s}$ & $P_{\min }^{n s}$ & {$\left[P^{n s} ; P^{-2 s} ; P^{+}\right]\left(\right.$Units of $\left.\frac{\pi}{M}\right)$} & $N$ & Tot \\
\hline 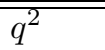 & 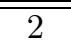 & 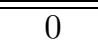 & "0 & 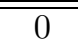 & $\overline{\bar{\pi} \pi / M}$ & 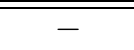 & $\overline{[-;-; 1,3]}$ & 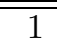 & 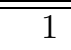 \\
\hline$q^{3}$ & 2 & 0 & 0 & 0 & $\pi / M$ & - & {$[-;-; 1,5]$} & 1 & 1 \\
\hline$q^{4}$ & 2 & 0 & 0 & 0 & $\pi / M$ & - & {$[-;-; 1,7],[-;-; 3,5]$} & 2 & 2 \\
\hline$q^{5}$ & 2 & 0 & 0 & 0 & $\pi / M$ & - & {$[-;-; 1,9],[-;-; 3,7]$} & 2 & 2 \\
\hline$q^{6}$ & 2 & 0 & 0 & 0 & $\pi / M$ & - & {$[-;-; 1,11],[-;-; 3,9],[-;-; 5,7]$} & 3 & \\
\hline \multirow[t]{2}{*}{$q^{7}$} & 2 & 0 & 0 & 0 & $\pi / M$ & - & {$[-;-; 1,13],[-;-; 3,11],[-;-; 5,9]$} & 3 & \\
\hline & 4 & 0 & 0 & 1 & $\pi / M$ & $-2 \pi / M$ & {$[-2 ;-; 1,3,5,7]$} & 1 & 4 \\
\hline \multirow[t]{2}{*}{$q^{8}$} & 2 & 0 & 0 & 0 & $\pi / M$ & - & $\begin{array}{l}{[-;-; 1,15],[-;-; 3,13],[-;-; 5,11]} \\
{[-;-; 7,9]}\end{array}$ & 4 & \\
\hline & 4 & 0 & 0 & 1 & $\pi / M$ & $-2 \pi / M$ & {$[-2 ;-; 1,3,5,9],[0,-, 1,3,5,7]$} & 2 & 6 \\
\hline \multirow[t]{3}{*}{$q^{9}$} & 2 & 0 & 0 & 0 & $\pi / M$ & - & {$[-;-; 1,17],[-;-; 3,15],[-;-; 5,13]$} & & \\
\hline & 4 & 0 & 0 & 1 & $\pi / M$ & $-2 \pi / M$ & {$[-2 ;-; 1,3,5,11],[-2 ;-; 1,3,7,9]$} & 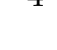 & \\
\hline & & & & & & & {$[0 ;-; 1,3,5,9],[2 ;-; 1,3,5,7]$} & 4 & 8 \\
\hline \multirow[t]{5}{*}{$q^{10}$} & 2 & 0 & 0 & 0 & $\pi / M$ & - & {$[-;-; 1,19],[-;-; 3,17],[-;-; 5,15]$} & & \\
\hline & & & & & & & {$[-;-; 7,13],[-;-; 9,11]$} & 5 & \\
\hline & 4 & 0 & 0 & 1 & $\pi / M$ & $-2 \pi / M$ & {$[-2 ;-; 1,3,5,13],[-2 ;-; 1,3,7,11]$} & & \\
\hline & & & & & & & {$[-2 ;-; 1,3,7,9],[0 ;-; 1,3,5,9]$} & & \\
\hline & 5 & 0 & 1 & 0 & 0 & - & $\begin{array}{l}{[0 ;-; 1,3,7,9],[2 ;-; 1,3,5,9]} \\
{[-:-; 0.2 .6 .8]}\end{array}$ & 0 & 12 \\
\hline \multirow[t]{8}{*}{$q^{11}$} & 2 & 0 & 0 & 0 & $\pi / M$ & - & {$[-;-; 1,21],[-;-; 3,19],[-;-; 5,17]$} & & \\
\hline & & & & & & & {$[-;-; 7,15],[-;-; 9,13]$} & 5 & \\
\hline & 4 & 0 & 0 & 1 & $\pi / M$ & $-2 \pi / M$ & {$[-2 ;-; 1,3,5,15],[-2 ;-; 1,3,7,13]$} & & \\
\hline & & & & & & & {$[-2 ;-; 1,5,7,11],[-2 ;-; 3,5,7,9]$} & & \\
\hline & & & & & & & {$[-2 ;-; 1,3,9,11],[0 ;-; 1,3,5,13]$} & & \\
\hline & & & & & & & {$[0 ;-; 1,3,7,11],[0 ;-; 1,5,7,9]$} & & \\
\hline & & & & & & & {$[2 ;-; 1,3,5,11],[2 ;-; 1,3,7,9]$} & 10 & \\
\hline & 5 & 0 & 1 & 0 & 0 & - & {$[-;-; 0,2,4,6,10]$} & 1 & 16 \\
\hline \multirow[t]{8}{*}{$q^{12}$} & 2 & 0 & 0 & $\overline{0}$ & $\pi / M$ & $\overline{-}$ & {$[-;-; 1,23],[-;-; 3,21],[-;-; 5,19]$} & & \\
\hline & 4 & 0 & 0 & 1 & $\pi / M$ & $-2 \pi / M$ & {$[-2 ;-; 1,3,5,17],[-2 ;-; 1,3,7,15]$} & & \\
\hline & & & & & & & {$[-2 ;-; 1,5,7,13],[-2 ;-; 3,5,7,11]$} & & \\
\hline & & & & & & & {$[-2 ;-; 1,3,9,13],[-2 ;-; 1,5,9,11]$} & & \\
\hline & & & & & & & {$[0 ;-; 1,3,5,15],[0 ;-; 1,3,7,13]$} & & \\
\hline & & & & & & & $\begin{array}{l}[0 ;-; 1,5,7,1]],[0 ;-; 3,5,7,9] \\
{[0 ;-; 1,3,9,11],[2 ;-; 1,3,5,13]}\end{array}$ & & \\
\hline & & & & & & & {$[2 ;-; 1,3,7,11],[2 ;-; 1,5,7,9]$} & 14 & \\
\hline & 5 & 0 & 1 & 0 & 0 & - & {$[-;-; 0,2,4,6,12],[-;-; 0,2,4,8,10]$} & 2 & 22 \\
\hline
\end{tabular}


Table 8: The first few terms in the sector of the partition function for the sector $Q=1$ and $m_{-}=m_{++}=0$, where $m_{+}=2 m_{n s}+3 m_{-}+4 m_{-2 s}$, and the momentum ranges are: $-\frac{\pi}{M}\left(m_{-2 s}+m_{-}-2\right) \leq P_{j}^{+}<\infty,-\frac{\pi}{M}\left(m_{-2 s}+m_{-}-2\right) \leq P_{j}^{-2 s} \leq \frac{\pi}{M}\left(m_{-2 s}+m_{-}-2\right),-\frac{\pi}{M}\left(m_{n s}+\right.$ $\left.2 m_{-2 s}+2 m_{-}-1\right) \leq P_{j}^{n s} \leq \frac{\pi}{M}\left(m_{n s}+2 m_{-2 s}+2 m_{-}-1\right)$.

\begin{tabular}{|c|c|c|c|c|c|c|c|c|c|}
\hline Order & $m_{+}$ & $m_{-2 s}$ & $m_{-}$ & $m_{n s}$ & $P_{\min }^{+,-2 s}$ & $P_{\min }^{n s}$ & {$\left[P^{n s} ; P^{-2 s} ; P^{+}\right]\left(\right.$Units of $\left.\frac{\pi}{M}\right)$} & $N$ & Tot \\
\hline 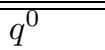 & 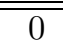 & 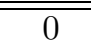 & $\overline{0}$ & 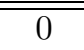 & - & - & {$[-;-;-]$} & 1 & $\overline{1}$ \\
\hline$q^{3}$ & 2 & 0 & 0 & 1 & $2 \pi / M$ & 0 & {$[0 ;-; 2,4]$} & 1 & 1 \\
\hline$q^{4}$ & 2 & $\overline{0}$ & $\overline{0}$ & 1 & $2 \pi / M$ & 0 & {$[0 ;-; 2,6]$} & 1 & 1 \\
\hline$q^{5}$ & 2 & 0 & 0 & 1 & $2 \pi / M$ & 0 & {$[0 ;-; 2,8],[0 ;-; 4,6]$} & 2 & 2 \\
\hline$q^{6}$ & 2 & 0 & 0 & 1 & $2 \pi / M$ & 0 & {$[0 ;-; 2,10],[0 ;-; 4,8]$} & 2 & 2 \\
\hline$q^{7}$ & 2 & $\overline{0}$ & 0 & 1 & $2 \pi / M$ & $\overline{0}$ & {$[0 ;-; 2,12],[0 ;-; 4,10],[0 ;-; 6,8]$} & 3 & 3 \\
\hline$q^{8}$ & 2 & 0 & 0 & 1 & $2 \pi / M$ & 0 & {$[0 ;-; 2,14],[0 ;-; 4,12],[0 ;-; 6,10]$} & 3 & 3 \\
\hline$q^{9}$ & 2 & 0 & 0 & 1 & $2 \pi / M$ & 0 & $\begin{array}{l}{[0 ;-; 2,16],[0 ;-; 4,14],[0 ;-; 6,12]} \\
{[0 ;-; 8,10]}\end{array}$ & 4 & 4 \\
\hline$q^{10}$ & 2 & $\overline{0}$ & 0 & 1 & $\begin{array}{l}2 \pi / M \\
2 \pi / M\end{array}$ & $\begin{array}{c}0 \\
-\pi / M\end{array}$ & $\begin{array}{l}{[0 ;-; 2,18],[0 ;-; 4,16],[0 ;-; 6,14]} \\
{[0 ;-; 8,12]} \\
{[-1,1 ;-; 2,4,6,8]}\end{array}$ & $\begin{array}{l}4 \\
1\end{array}$ & 5 \\
\hline$q^{11}$ & 2 & 0 & 0 & 1 & $\begin{array}{l}2 \pi / M \\
2 \pi / M\end{array}$ & $\begin{array}{c}0 \\
-\pi / M\end{array}$ & $\begin{array}{l}{[0 ;-; 2,20],[0 ;-; 4,18],[0 ;-; 6,16]} \\
{[0 ;-; 8,14],[0 ;-; 10,12]} \\
{[-1,1 ;-; 2,4,6,10]}\end{array}$ & $\begin{array}{l}5 \\
1\end{array}$ & 6 \\
\hline$q^{12}$ & 2 & 0 & 0 & 1 & $\begin{array}{l}2 \pi / M \\
2 \pi / M\end{array}$ & $\begin{array}{c}0 \\
-\pi / M\end{array}$ & $\begin{array}{l}{[0 ;-; 2,22],[0 ;-; 4,20],[0 ;-; 6,18]} \\
{[0 ;-; 8,16],[0 ;-; 10,14]} \\
{[-1,1 ;-; 2,4,6,12],[-1,1 ;-; 2,4,8,10]}\end{array}$ & $\begin{array}{l}5 \\
2\end{array}$ & 7 \\
\hline
\end{tabular}


Table 9: The first few terms for the sector of the partition function corresponding to $Q=1$ and $m_{-}=m_{++} \neq 0$, where $m_{+}=2 m_{n s}+3 m_{-}+4 m_{-2 s}$, and the momentum ranges are: $-\frac{\pi}{M}\left(m_{-2 s}+m_{-}-3\right) \leq P_{j}^{+}<\infty,-\frac{\pi}{M}\left(m_{-2 s}+m_{-}-1\right) \leq P_{j}^{-2 s} \leq \frac{\pi}{M}\left(m_{-2 s}+m_{-}-3\right)$, $-\frac{\pi}{M}\left(m_{n s}+2 m_{-2 s}+2 m_{-}\right) \leq P_{j}^{n s} \leq \frac{\pi}{M}\left(m_{n s}+2 m_{-2 s}+2 m_{-}-2\right)$, and there is a shift of $-\frac{2 \pi}{M}\left(\frac{1}{2} m_{n s}+m_{-2 s}+m_{-}\right)$.

\begin{tabular}{|c|c|c|c|c|c|c|c|c|c|c|}
\hline Order & $m_{+}$ & $m_{-2 s}$ & $m_{-}$ & $m_{n s}$ & $P_{\min }^{+,-2 s}$ & $P_{\min }^{n s}$ & {$\left[P^{n s} ; P^{-2 s} ; P^{+}\right]\left(\right.$Units of $\left.\frac{\pi}{M}\right)$} & Shift & $\mathrm{N}$ & Tot \\
\hline$\overline{\overline{q^{5}}}$ & 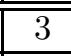 & $\overline{0}$ & 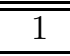 & 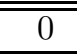 & $2 \pi / M$ & 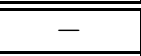 & 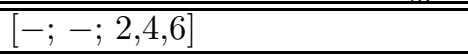 & $-2 \pi / M$ & 1 & 1 \\
\hline$q^{6}$ & 3 & 0 & 1 & 0 & $2 \pi / M$ & - & {$[-;-; 2,4,8]$} & $-2 \pi / M$ & 1 & 1 \\
\hline$q^{7}$ & 3 & 0 & 1 & 0 & $2 \pi / M$ & - & {$[-;-; 2,4,10],[-;-; 2,6,8]$} & $-2 \pi / M$ & 2 & $\overline{2}$ \\
\hline$q^{8}$ & 3 & 0 & 1 & $\overline{0}$ & $2 \pi / M$ & - & $\begin{array}{l}{[-;-; 2,4,12],[-;-; 2,6,10]} \\
{[-;-; 4,6,8]}\end{array}$ & $-2 \pi / M$ & 3 & 3 \\
\hline$q^{9}$ & 3 & 0 & 1 & 0 & $2 \pi / M$ & - & $\begin{array}{l}{[-;-; 2,4,14],[-;-; 2,6,12]} \\
{[-;-; 4,6,10],[-;-; 2,8,10]}\end{array}$ & $-2 \pi / M$ & 4 & 4 \\
\hline$q^{10}$ & 3 & 0 & 1 & $\overline{0}$ & $2 \pi / M$ & - & $\begin{array}{l}{[-;-; 2,4,16],[-;-; 2,6,14]} \\
{[-;-; 4,6,12],[-;-; 2,8,12]} \\
{[-;-; 4,8,10]}\end{array}$ & $-2 \pi / M$ & 5 & 5 \\
\hline$q^{11}$ & 3 & 0 & 1 & 0 & $2 \pi / M$ & - & $\begin{array}{l}{[-;-; 2,4,18],[-;-; 2,6,16]} \\
{[-;-; 4,6,14],[-;-; 2,8,14]} \\
{[-;-; 4,8,12],[-;-; 6,8,10]} \\
{[-;-; 2,10,12]}\end{array}$ & $-2 \pi / M$ & 7 & 7 \\
\hline$q^{12}$ & 3 & 0 & 1 & 0 & $\begin{array}{l}2 \pi / M \\
2 \pi / M \\
\end{array}$ & $-3 \pi / M$ & $\begin{array}{l}{[-;-; 2,4,20],[-;-; 2,6,18]} \\
{[-;-; 4,6,16],[-;-; 2,8,16]} \\
{[-;-; 4,8,14],[-;-; 6,8,12]} \\
{[-;-; 2,10,12],[-;-; 4,10,12]} \\
{[-3 ;-; 2,4,6,8,10]}\end{array}$ & $\begin{array}{l}-2 \pi / M \\
-3 \pi / M \\
\end{array}$ & $\begin{array}{l}8 \\
1 \\
\end{array}$ & 9 \\
\hline
\end{tabular}


Table 10: The first few terms for the sector of the partition function corresponding to $Q=1$ and $m_{-}=m_{++} \neq 0$, where $m_{+}=2 m_{n s}+3 m_{-}+4 m_{-2 s}$, and the momentum ranges are: $-\frac{\pi}{M}\left(m_{-2 s}+m_{-}-1\right) \leq P_{j}^{+}<\infty,-\frac{\pi}{M}\left(m_{-2 s}+m_{-}-1\right) \leq P_{j}^{-2 s} \leq \frac{\pi}{M}\left(m_{-2 s}+m_{-}-3\right),-\frac{\pi}{M}\left(m_{n s}+\right.$ $\left.2 m_{-2 s}+2 m_{-}-2\right) \leq P_{j}^{n s} \leq \frac{\pi}{M}\left(m_{n s}+2 m_{-2 s}+2 m_{-}\right)$. There is an additive shift in the total momentum of $\frac{2 \pi}{M}\left(\frac{1}{2} m_{n s}+m_{-2 s}+m_{-}\right)$.

\begin{tabular}{|c|c|c|c|c|c|c|c|c|c|c|}
\hline Order & $m_{+}$ & $m_{-2 s}$ & $m_{-}$ & $m_{n s}$ & $P_{\min }^{+,-2 s}$ & $P_{\min }^{n s}$ & {$\left[P^{n s} ; P^{-2 s} ; P^{+}\right]\left(\right.$Units of $\left.\frac{\pi}{M}\right)$} & Shift & $N$ & Tot \\
\hline 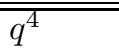 & 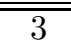 & 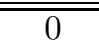 & 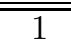 & 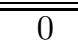 & 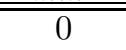 & $\overline{-}$ & $\overline{[-;}-; 0,2,4]$ & $2 \pi / M$ & 1 & $\overline{1}$ \\
\hline$q^{5}$ & 3 & 0 & 1 & 0 & 0 & - & {$[-;-; 0,2,6]$} & $2 \pi / M$ & 1 & 1 \\
\hline$q^{6}$ & 3 & 0 & 1 & 0 & 0 & - & {$[-;-; 0,2,8],[-;-; 0,4,6]$} & $2 \pi / M$ & 2 & 2 \\
\hline$q^{7}$ & 3 & 0 & 1 & 0 & 0 & $\overline{-}$ & $\begin{array}{l}{[-;-; 0,2,10],[-;-; 0,4,8]} \\
{[-;-; 2,4,6]}\end{array}$ & $2 \pi / M$ & 3 & 3 \\
\hline$q^{8}$ & 3 & 0 & 1 & 0 & 0 & - & $\begin{array}{l}{[-;-; 0,2,12],[-;-; 0,4,10]} \\
{[-;-; 2,4,8],[-;-; 0,6,8]}\end{array}$ & $2 \pi / M$ & 4 & 4 \\
\hline$q^{9}$ & 3 & 0 & 1 & 0 & 0 & - & $\begin{array}{l}{[-;-; 0,2,14],[-;-; 0,4,12]} \\
{[-;-; 2,4,10],[-;-; 0,6,10]} \\
{[-;-; 2,6,8]}\end{array}$ & $2 \pi / M$ & 5 & 5 \\
\hline$q^{10}$ & 3 & 0 & 1 & 0 & 0 & - & $\begin{array}{l}{[-;-; 0,2,16],[-;-; 0,4,14]} \\
{[-;-; 2,4,12],[-;-; 0,6,12]} \\
{[-;-; 2,6,10],[-;-; 4,6,8]} \\
{[-;-; 0,8,10]}\end{array}$ & $2 \pi / M$ & 7 & 7 \\
\hline$q^{11}$ & 3 & 0 & 1 & 0 & 0 & $-\pi / M$ & $\begin{array}{l}{[-;-; 0,2,18],[-;-; 0,4,16]} \\
{[-;-; 2,4,14],[-;-; 0,6,14]} \\
{[-;-; 2,6,12],[-;-; 4,6,10]} \\
{[-;-; 0,8,12],[-;-; 2,8,10]} \\
{[-1 ;-; 0,2,4,6,8]}\end{array}$ & $\begin{array}{l}2 \pi / M \\
3 \pi / M\end{array}$ & $\begin{array}{l}8 \\
1\end{array}$ & 9 \\
\hline$q^{12}$ & 3 & 0 & 1 & 0 & 0 & $-\pi / M$ & $\begin{array}{l}{[-;-; 0,2,20],[-;-; 0,4,18]} \\
{[-;-; 2,4,16],[-;-; 0,6,16]} \\
{[-;-; 2,6,14],[-;-; 4,6,12]} \\
{[-;-; 0,8,14],[-;-; 2,8,12]} \\
{[-;-; 0,10,12],[-;-; 4,8,10]} \\
{[-1 ;-; 0,2,4,6,10],} \\
{[1 ;-; 0,2,4,6,8]}\end{array}$ & $\begin{array}{l}2 \pi / M \\
3 \pi / M\end{array}$ & $\begin{array}{r}10 \\
2\end{array}$ & 12 \\
\hline
\end{tabular}

